4 Manuel Brenes-Álvarez, Marina Minguet ${ }^{\dagger}$, Agustín Vioque*, and Alicia M. Muro-Pastor

NsiR1, a small RNA with multiple copies, modulates heterocyst differentiation in the cyanobacterium Nostoc sp. PCC 7120

7 Instituto de Bioquímica Vegetal y Fotosíntesis, Consejo Superior de

8 Investigaciones Científicas and Universidad de Sevilla, Sevilla, Spain.

9

$10{ }^{\dagger}$ Present address: Centro de Biología Molecular Severo Ochoa, CSIC-UAM,

11 Nicolas Cabrera 1, 28049, Madrid, Spain.

12

.

6

*Corresponding author: Agustín Vioque, Instituto de Bioquímica Vegetal y

4 Fotosíntesis, Américo Vespucio 49, 41092, Sevilla, Spain

Phone: +34-954489519; Email: vioque@us.es

21 Running title: sRNA regulation of heterocyst differentiation

\title{
Keywords
}

24 Anabaena, HetF, HetR, sRNA, post-transcriptional regulation 


\section{Originality-significance statement:}

Heterocysts are nitrogen-fixing specialized cells that appear at semi-regular intervals along cyanobacterial filaments upon nitrogen starvation. Heterocyst differentiation and patterning is a model system to study cell differentiation in multicellular prokaryotes. The differentiation process has a complex regulation, only partially understood, that includes transcriptional changes, factor diffusion between cells, and protein-protein interactions. This work adds to this complexity a small non-coding RNA, NsiR1, that negatively regulates at a posttranscriptional level the expression of hetF, a gene that is part of the heterocyst differentiation regulatory network. Thus, regulation by a small RNA is a new element involved in the control of heterocyst differentiation. A better understanding of the regulation of heterocyst differentiation could be useful for

42 the engineering of biological $\mathrm{N}_{2}$ fixation or the production of $\mathrm{O}_{2}$-sensitive molecules in cyanobacteria. 
47 Upon nitrogen starvation filamentous cyanobacteria develop heterocysts, specialized cells devoted to fixation of atmospheric nitrogen. Differentiation of heterocyst at semi-regular intervals along the filaments requires complex structural and functional changes that are under control of the master transcriptional regulator HetR. NsiR1 (nitrogen stress-induced RNA $\underline{1}$ ) is a HetR-dependent non-coding RNA that is expressed specifically in heterocysts from a very early stage of differentiation. In the genome of Nostoc sp. PCC 7120 there are twelve tandem copies of $n \operatorname{siR} 1$ ( $n \operatorname{siR} 1.1$ to $n \operatorname{siR} 1.12$ ), seven of

55 them with identical sequence ( $n \operatorname{siR} 1.3$ to $n \operatorname{siR} 1.9)$ and the others slightly

56 divergent. $n s i R 1.1$ is transcribed antisense to the 5' UTR of hetF, a gene

57 required for heterocyst development. Here we show that binding of NsiR1.1 inhibits translation of the hetF mRNA by inducing structural changes in its 5 ' UTR. Altered levels of NsiR1 result in different phenotypic alterations including

60 enlarged cell size and delayed heterocyst development that could be related to a reduced amount of HetF.

62 
Introduction

Cyanobacteria are the only prokaryotes able to perform oxygenic photosynthesis and important contributors to global primary productivity. They constitute a coherent phylogenetic group with diverse morphology and wide ecological distribution. Nostoc sp. PCC 7120 (a.k.a Anabaena sp. PCC 7120) is a filamentous cyanobacterium that under nitrogen deprivation differentiates heterocysts, specialized cells devoted to fixation of atmospheric nitrogen. In the absence of combined nitrogen, filaments are composed of photosynthetic vegetative cells and regularly spaced (every 10-15 cells) nitrogen fixing heterocysts. Heterocyst differentiation involves a complex developmental program (Flores and Herrero, 2010; Muro-Pastor and Hess, 2012; Herrero et al., 2016; Flores et al., 2019) that is ultimately under control of NtcA, the global regulator of nitrogen assimilation (Herrero et al., 2001), but also under control of HetR, a specific regulator of cell differentiation (Buikema and Haselkorn, 1991; Black et al., 1993). At the initiation of heterocyst differentiation, induction of the expression of NtcA and HetR depends on each other (Muro-Pastor et al., 2002) and leads to increased expression of HetR in those cells becoming heterocysts. Expression of hetR is regulated at several levels. hetR is transcribed from a complex promoter including several transcriptional start sites (TSSs), one of them specifically induced in cells becoming heterocysts (Rajagopalan and Callahan, 2010; Mitschke et al., 2011). HetR is a DNA-binding protein that positively regulates its own expression and the expression of many genes, including negative elements essential for the maintenance of the pattern of heterocysts along the filaments, such as patS (Yoon and Golden, 1998), hetN 
(Callahan and Buikema, 2001) and patX (Elhai and Khudyakov, 2018). The binding of the pentapeptide RGSGR, that is part of the sequence of PatS, blocks the DNA-binding activity of HetR (Huang et al., 2004) and signals its degradation (Risser and Callahan, 2009). HetR is an autoproteolytic protein (Zhou et al., 1998) that seems also to be degraded by the HetF protease (Wong and Meeks, 2001; Risser and Callahan, 2008). A hetF mutant accumulates a large amount of HetR, has an altered morphology with enlarged cells and does not differentiate heterocysts (Risser and Callahan, 2008), suggesting HetR turnover is required for differentiation. However, since hetF transcription seems unpatterned along the filaments (Risser and Callahan, 2008), the question of how HetF may contribute to the differential accumulation of active HetR in heterocysts versus vegetative cells remains open.

Small non-coding RNAs (sRNAs) are important regulators of gene expression in bacteria, including cyanobacteria (Kopf and Hess, 2015; Muro-Pastor and Hess, 2020). Most frequently they bind to their target mRNAs close to the ribosome binding site therefore inhibiting translation, but a large diversity of alternative regulatory mechanisms operated by sRNAs are known (Wagner and Romby, 2015). They are involved in the regulation of diverse processes related to response to stress or adaptation to a changing environment, including cell differentiation. In Bacillus several sRNAs have been identified that are sporulation-specific and with expression restricted to the forespore (Silvaggi et al., 2006; Schmalisch et al., 2010; Marchais et al., 2011; Mars et al., 2016). In heterocyst-forming cyanobacteria, sRNAs and antisense RNAs with heterocystspecific expression have been identified (lonescu et al., 2010; Brenes-Álvarez et al., 2016; Brenes-Álvarez et al., 2019). A heterocyst-specific antisense RNA 
114 is involved in carbon fixation shutdown in heterocysts (Olmedo-Verd et al., 115 2019).

116 NsiR1 is a sRNA of about 60 nucleotides encoded in 12 tandem copies in the 117 region upstream of hetF in Nostoc sp. PCC 7120 (lonescu et al., 2010) (Fig.

118 1A). NsiR1 is expressed specifically in cells becoming heterocysts before any 119 sign of morphological differentiation is observed (Muro-Pastor, 2014).

120 Transcription of the different copies of NsiR1 requires HetR (lonescu et al., 2010) and takes place from individual promoters that contain the DIF1 sequence motif associated to heterocyst-specific transcription (Mitschke et al., 2011; Brenes-Álvarez et al., 2019). It is currently unknown whether transcription of NsiR1 is regulated by HetR directly or indirectly. The central seven copies $n \operatorname{siR} 1.3$ to $n \operatorname{siR} 1.9$ (thereafter referred to as $n \operatorname{siR} 1.4$ ) are identical whereas the other copies, located at the periphery of the array, have some sequence divergence. According to the position of the TSS of hetF (Ionescu et al., 2010; Mitschke et al., 2011), nsiR1.1 overlaps the hetF 5' UTR. NsiR1.1 could therefore function as an antisense of the hetF transcript. As the seven identical copies are very similar to nsiR1.1, they could also potentially regulate the could have additional non-overlapping targets.

Results 
140 NsiR1.1 is transcribed antisense to the 257 nt long 5' UTR of hetF in Nostoc sp.

PCC 7120 and this arrangement is conserved among genomes of

heterocystous cyanobacteria (lonescu et al., 2010) (Supporting Information Fig.

S1 and S2), therefore we hypothesized that NsiR1.1 might regulate hetF

expression. If NsiR1 binding to hetF 5' UTR regulates hetF expression, we

would expect different accumulation of HetF protein in heterocysts versus vegetative cells because nsiR1 transcription is heterocyst-specific (lonescu et al., 2010; Brenes-Álvarez et al., 2019). To test this hypothesis, we constructed Nostoc sp. PCC 7120 strains that express, under the control of a constitutive promoter $(r n p B)$, a translational fusion of the hetF 5' UTR plus 60 nucleotides of its coding region to gfpmut2 (plasmid pMBA93, see a scheme in Fig. 1A). A strain expressing gfpmut2 and its original 5'UTR from the rnpB promoter was constructed as a control (plasmid pMBA92, Fig. 1A). As expected the strain bearing the control plasmid pMBA92 showed similar green fluorescence in vegetative cells or heterocysts (Fig. 1B). However, the strain bearing pMBA93 showed detectable green fluorescence in vegetative cells but not in heterocysts (Fig. 1B), suggesting heterocyst-specific downregulation at the level of the hetF 5' UTR.

We then tested the interaction of NsiR1.1 with hetF 5' UTR using a heterologous reporter assay in E. coli (Urban and Vogel, 2007; Corcoran et al., 2012) (see Materials and Methods for details) in which the 5' UTR of hetF plus 60 nucleotides of its coding region was fused to the gene sfgfp encoding superfolder GFP (sfGFP). Because identical copies (nsiR1.3 to $n \operatorname{siR1.9)}$ ) are 
very similar to $n s i R 1.1$ (lonescu et al., 2010), we also tested the interaction between the hetF 5' UTR and the sRNA encoded in one of the identical copies, namely nsiR1.4 (Supporting Information Fig. S3). We therefore co-expressed in E. coli the hetF::sfgfp fusion together with NsiR1.1, NsiR1.4 or a control nonsense RNA corresponding to the unrelated terminator of the $\operatorname{rrn} B$ gene of $E$. coli (transcribed from plasmid pJV300, see Materials and Methods). For this experiment both the hetF::sfgfp translational fusion and the different RNAs are transcribed constitutively. Cells carrying the hetF::sfgfp translational fusion showed significant fluorescence, indicating that the translational initiation region of hetF was functional in $E$. coli (Fig. 1C and D). The fluorescence of cells carrying the hetF::sfgfp fusion decreased about $70 \%$ or $40 \%$ of the control when the fusion was co-expressed with NsiR1.1 or NsiR1.4, respectively, suggesting that each of these versions of NsiR1 was able to interact with the 5' UTR of hetF (Fig. 1C and D) when transcribed in a heterologous system.

Taken together, the results obtained in Nostoc sp. PCC 7120 and E. coli suggested a post-transcriptional negative regulation exerted by NsiR1 on hetF expression. The overlapping of NsiR1.1 and the hetF 5' UTR (Fig. 1A) extends between positions 20 and 78 of the UTR, 180 nucleotides upstream the start codon. To analyze the possible regulatory mechanism, we carried out in vitro footprinting assays of the interaction between in vitro transcribed NsiR1.1 and hetF 5' UTR (Fig. 2). ${ }^{32} \mathrm{P}-$ labeled hetF 5' UTR (a fragment extending from the TSS at position -257 to +60 with respect to the start of the coding sequence) was incubated with unlabeled NsiR1.1 and probed with RNase T1, RNase A, or lead(II) acetate (Fig. 2A). A footprint was detected between positions 23 and 81 of hetF 5' UTR (Fig. 2A, red bar), in agreement with the perfect antisense 
sequence complementary to NsiR1.1. In addition to this footprint, we also detected some structural changes when hetF 5' UTR was combined with NsiR1.1; a region between positions 98 and 104 of hetF 5' UTR became more sensitive to RNase T1, RNase A and lead(II) acetate (Fig. 2A, green bar) and the region including the start codon became less accessible (Fig. 2A, orange bar). These results suggest that upon binding of NsiR1 there is a structural reorganization of the $5^{\prime}$ UTR of hetF resulting in reduced access to the initiation codon (Fig. 2B and C) that could explain the reduction of HetF expression in heterocysts.

\section{Construction of strains with altered levels of different versions of NsiR1}

In order to analyze possible phenotypic effects caused by altered levels of NsiR1, we have constructed strains with constitutive high levels of NsiR1.1 (OE_NsiR1.1) or NsiR1.4 (OE_NsiR1.4) transcribed from the strong trc promoter to the $T 1$ terminator of the $r r n B$ gene of $E$. coli (Fig. 3A). In addition, because we have introduced a Nsil restriction site (6 bp) at the 5' end of $n \operatorname{siR} 1.1$ or $n \operatorname{siR} 1.4$ to facilitate cloning, this $6 \mathrm{nt}$ tag allows the discrimination, based on its length, of molecules of NsiR1.1 and NsiR1.4 transcribed from their native promoters from molecules expressed from the trc promoter in the constructs introduced in Nostoc sp. PCC 7120.

The location of $n$ siR1.1 in the 5' UTR of hetF precludes the deletion of all copies of $n$ siR1 without removing a segment of hetF 5' UTR. As an alternative strategy to deplete NsiR1 without altering the genomic region upstream of hetF we have followed a strategy similar to that in Brenes-Álvarez et al., (2020) and generated 
a Nostoc strain (OE_as_NsiR1) that constitutively expresses the sequence complementary to nsiR1.4 from the trc promoter (Fig. 3A). This antisense RNA is expected to act as a sponge neutralizing the accumulation of $n s i R 1$. Using probes specific for NsiR1.1 or NsiR1.4 we have verified by Northern blot the constitutive expression of NsiR1.1 and NsiR1.4 under the control of the trc promoter in strains OE_NsiR1.1 and OE_NsiR1.4, respectively (Fig. 3B). In addition, Northern blot hybridization also showed that, in the OE_C strain, signals corresponding to hybridization to the probe for NsiR1.4 (corresponding to transcription of endogenous $n \operatorname{siR} 1.3$ to $n \operatorname{siR} 1.9)$ were stronger than those corresponding to $n s i R 1.1$, as expected from the different number of identical copies of $n \operatorname{siR} 1.4$ (seven copies) versus $n \operatorname{siR} 1.1$ (single copy). Transcripts corresponding to NsiR1.1 and NsiR1.4 whose transcription did not stop at their own Rho-independent terminators but instead were terminated at the $T 1$ terminator were also observed above the $100 \mathrm{nt}$ long size marker (indicated hybridization showed that the accumulation of NsiR1.1 and NsiR1.3 to NsiR1.9 was diminished in this strain, suggesting effective removal of endogenous native NsiR1 by overexpression of the antisense sponge (Fig. 3B). Both probes used for hybridization included the T1 terminator, present also in as_NsiR1 (Fig. 3A), leading to its detection in the OE_as_NsiR1 strain (Fig. 3B).

We were unable to quantify hetF expression by Northern blot or RT-PCR, suggesting that, similar to the observations previously made in Nostoc punctiforme (Wong and Meeks, 2001), the hetF transcript is likely unstable or accumulates in small quantity in Nostoc sp. PCC 7120. We have instead analyzed the accumulation of hetF transcripts and their putative interaction with 
239 the different versions of NsiR1 by primer extension assays (Fig. 3C). The 5'ends of hetF transcripts were analyzed by retrotranscription using an oligonucleotide complementary to hetF 5' UTR. Extension products corresponding to the TSS of hetF, position $4273166 f$ in the Nostoc sp. PCC 7120 genome (lonescu et al., 2010; Mitschke et al., 2011), were in fact observed using RNA samples isolated from wild type strain or the hetR mutant (Supporting Information Fig. S4). In addition, shorter HetR-dependent extension products were also observed around position 4173242, corresponding to the TSS of $n$ siR1.1 (Supporting Information Fig. S4). Since, according to dRNA-Seq (Mitschke et al 2011), there are no additional TSS for hetF in this region, these shorter extension products could be the result of termination of retrotranscription at processing sites or regions with strong secondary structure. Similar to the observation made in the wild-type strain (Supporting Information Fig. S4), extension products that would correspond to the full-length 5' UTR of hetF were observed using RNA from the control strain OE_C as well as from strains OE_NsiR1.4 and OE_as_NsiR1 (indicated with an asterisk in Fig. 3C) but not in strain OE_NsiR1.1 (see below). Extension products that stopped at the position corresponding to the TSS of the chromosomal nsiR1.1 were observed using RNA isolated from the OE_C, OE_NsiR1.1 and OE_NsiR1.4 strains when the cells were subjected to nitrogen deficiency (Fig. 3C, black nitrogen deficiency and in reduced amounts. Retrotranscriptase stops when it encounters an obstacle, for example, regions of strong secondary structure or a double stranded RNA such as a sRNA-mRNA duplex (Jagodnik et al., 2017). 
264 Because of the location of the additional stops observed, one possible explanation is that they are produced by binding of NsiR1. In fact, and in contrast to the other three strains included in the experiment, no full-length extension products that corresponded to the 5'-end of hetF were observed using RNA isolated from strain OE_NsiR1.1. Instead, a strong stop around the start position of nsiR1.1 was observed irrespective of nitrogen removal (Fig. 3C, green triangle), suggesting that the presence of NsiR1.1 expressed from the constitutive trc promoter was responsible for such stops. Similarly, stops independent of the nitrogen condition but weaker than those observed with RNA isolated from strain OE_NsiR1.1 were also observed with RNA isolated from the OE_NsiR1.4 strain (Fig. 3C, red triangle). Taken together, these observations indicate that the HetR-dependent NsiR1.1 sRNA (and possibly NsiR1.4) interacts with the 5' UTR of hetF in Nostoc sp. PCC 7120.

Based on data from fluorescence microscopy in Nostoc strains bearing pMBA93, the observations made in E. coli, and in vitro footprinting assays (Fig. regulation of hetF expression. Because it is known that a hetF mutant accumulates more HetR protein than the WT strain (Risser and Callahan, 2008), we quantified the accumulation of HetR in the OE_C, OE_NsiR1.1, OE_NsiR1.4 and OE_as_NsiR1 strains $18 \mathrm{~h}$ after combined nitrogen removal as an indication of HetF levels (Fig. 3D and E). Using three independent clones of each strain, we determined that strain OE_NsiR1.1 accumulated about twice more HetR protein than the OE_C strain which could be a consequence of reduced HetF amounts in OE_NsiR1.1. OE_NsiR1.4 also seems to accumulate more HetR protein than the OE_C strain, but in this case, perhaps due to the 
effects of NsiR1.4 on HetF accumulation being weaker, the results were not quite statistically significant $(p=0.057)$. In order to analyze whether the increased amounts of HetR protein could be instead due to increased transcription of hetR in the overexpressing strains, we measured the accumulation of hetR transcripts in all four strains analyzed above (OE_C,

OE_NsiR1.1, OE_NsiR1.4 and OE_as_NsiR1) (Supporting Information Fig. S5).

After $18 \mathrm{~h}$ of nitrogen deficiency the amount of hetR transcript in the

OE_NsiR1.1 strain was not above the amounts observed in the control strain

$\left(\mathrm{OE} \_\mathrm{C}\right)$, indicating that the increased amount of HetR in strain OE_NsiR1.1 was not the consequence of increased transcription of the hetR gene.

Altered heterocyst differentiation in strains OE_NsiR1.1, OE_NsiR1.4 and OE_as_NsiR1

302

In order to analyze the effects of altered NsiR1 expression at the phenotypic level we tested growth of the OE_C, OE_NsiR1.1, OE_NsiR1.4 and OE_as_NsiR1 strains in solid media containing different nitrogen sources (Fig. 4A). All four strains grew similarly in the presence of ammonium, however strain OE_NsiR1.4 grew worse in the absence of combined nitrogen and a bit slower in the presence of nitrate than the rest of strains. In liquid cultures, strain OE_NsiR1.4 showed slight phenotypic differences (larger cell size) in the presence of ammonium. The differences were more dramatic after combined nitrogen removal (Fig. 4B). After nitrogen removal, strain OE_NsiR1.4 differentiated cells that were stained by Alcian blue (indicative of the presence of heterocyst polysaccharides) but some filaments had enlarged cells which 
resembled the phenotype previously described for the hetF mutant (Risser and

315 Callahan, 2008). OE_NsiR1.1, also contained some filaments with larger cells under nitrogen deprivation. Frequency of heterocysts in filaments of strains with altered levels of NsiR1 was determined taking samples at different time points after nitrogen removal and staining with Alcian blue. Whereas all four strains reached similar frequency of heterocyst after 72 hours of nitrogen deprivation, different frequencies were observed at an earlier time point (24 h) indicating a delay in differentiation of heterocysts in strains OE_NsiR1.1 and OE_as_NsiR1 (Fig. 4C). This delay was longer in OE_as_NsiR1, the only strain with a statistically significant difference in frequency of heterocyst after 48 hours (Fig. $4 C)$.

Nitrogen fixation is carried out by nitrogenase, and expression of the nifHDK genes can be taken as an indication of heterocyst maturity. We therefore analyzed the timing of expression of nifH during heterocyst differentiation upon nitrogen removal in the different strains (Fig. 4D). OE_NsiR1.1 and OE_NsiR1.4 showed no difference with respect to the control, however OE_as_NsiR1, depleted of NsiR1, showed a delay in the accumulation of nifH mRNA, suggesting a delay in differentiation of mature heterocysts.

\section{Discussion}

Heterocyst differentiation is a complex process that requires precise regulatory mechanisms in order to establish exclusive transcriptional programs for different cell types (vegetative cells, immature pro-heterocysts and mature heterocysts). 
Heterocyst differentiation is triggered by mutual induction of expression of the transcriptional regulator NtcA and the master regulator HetR in vegetative cells becoming heterocysts (Black et al., 1993; Muro-Pastor et al., 2002; OlmedoVerd et al., 2006). The accumulation of active HetR in pro-heterocysts seems to trigger the downstream cascade. NsiR1 was the first HetR-dependent sRNA identified in Nostoc sp. PCC 7120 (Ionescu et al., 2010), but it is also well conserved in non-heterocyst-forming filamentous cyanobacteria (BrenesÁlvarez et al., 2016). However, while most non-heterocystous strains contain a single copy of NsiR1, heterocystous strains have multiple tandem copies with slight sequence divergence (Supporting Information Fig. S1, S2 and S6). The increase in the number of copies of NsiR1, that correlates with the evolution of the ability to differentiate heterocysts, and their sequence divergence, could allow some degree of functional specialization by regulating a variety of targets. According to the position of the TSS of hetF in Nostoc sp. PCC 7120 (Ionescu et al., 2010; Mitschke et al., 2011), the nsiR1 copy closest to hetF (nsiR1.1) could function as an antisense of hetF 5' UTR. This function of NsiR1.1 may be conserved in heterocyst-forming cyanobacteria because most of them have a $n s i R 1$ homolog in the upstream region of hetF in antisense orientation (Supporting Information Fig. S1 and S2).

Co-expression experiments in E. coli indicate that NsiR1.1, and to a lower extent NsiR1.4, can repress the expression of hetF (Fig. 1C and D). A translational fusion of hetF 5' UTR to GFP had reduced expression in heterocysts (Fig. 1B), as expected if its translation is repressed by NsiR1, that is expressed at an early stage during heterocyst development (Muro-Pastor, 2014). In addition, primer extension analysis in Nostoc sp. PCC 7120 also 
suggested a putative interaction of NsiR1.1 with hetF 5' UTR based on the stop of retrotranscription (Supporting Information Fig. S4). Several observations point to post-transcriptional regulation of translation rather than transcriptional interference as the mechanism for hetF regulation by NsiR1. First, negative regulation of hetF expression is observed in the E. coli system, in which NsiR1 and the hetF 5' UTR are constitutively transcribed from E. coli promoters in separate plasmids. Second, the observation that NsiR1.1, which perfectly matches the hetF 5' UTR, and NsiR1.4 which contains several nucleotide changes, repress hetF translation to a different extent (Fig. 1C), is indicative of post-transcriptional regulation by perfect (NsiR1.1) or imperfect (NsiR1.4) base pairing. Finally, the total quantity of hetF mRNA did not significantly decrease when NsiR1 was present (Supporting Information Fig. S4), indicating that NsiR1 did not affect hetF transcription.

The interaction site of NsiR1 in the hetF 5' UTR was located relatively far upstream from the hetF start codon, precluding repression of translation by direct interaction of NsiR1 with the translation initiation region and pointing instead to a translational regulation based on a possible conformational change of the hetF 5' UTR. In vitro footprinting data suggest that the hetF 5' UTR is highly structured and the interaction with NsiR1.1 provokes a conformational change in which the region of the start codon of hetF becomes less accessible (Fig. 2), providing a mechanistic explanation for the negative regulation of hetF expression by NsiR1.1.

In order to study the function of NsiR1, we created strains of Nostoc sp. PCC 7120 with altered levels of different versions of NsiR1 (Fig. 3). The overexpression of NsiR1.1 or NsiR1.4 under the control of the trc promoter was 
irrespective of the nitrogen source and strong enough to overpass the endogenous expression of NsiR1, that was induced upon nitrogen removal. As expected, the OE_as_NsiR1 strain had reduced amounts of NsiR1, implying that the interaction between NsiR1 and its antisense results in its degradation (Fig. 3B). Primer extension assays with RNA extracted from strains with altered levels of different versions of NsiR1 confirmed the interaction of NsiR1.1 and NsiR1.4 with hetF 5' UTR (Fig. 3C). We were unable to quantify changes in hetF expression in our mutant strains, likely because expression of hetF is low and the transcripts are unstable (Wong and Meeks, 2001; Risser and Callahan, 2008). Instead, because one of the features described for a hetF mutant is the accumulation of higher levels of HetR protein (Risser and Callahan, 2008), we analyzed HetR protein levels as an indication of HetF function. In fact, strains OE_NsiR1.1 and, to a lesser extent, OE_NsiR1.4, had higher amounts of HetR, as expected for a strain with a reduced amount of HetF (Fig. 3D and E). This higher amount of HetR was not due to increased hetR transcription in strains OE_NsiR1.1 and OE_NsiR1.4 (Supporting Information Fig. S5). Finally, OE_NsiR1.1 had a phenotype partially similar in some respects to that described for a hetF mutant. A hetF mutant grows poorly in media without combined nitrogen, has enlarged cells and cannot differentiate heterocysts (Risser and Callahan, 2008). OE_NsiR1.1 showed some filaments with larger cells in liquid cultures growing without combined nitrogen (Fig. 4). Taken together, all these in vivo and in vitro results strongly support a role for NsiR1 in reducing the amount of HetF in cells becoming heterocysts. Proper HetR turnover seems to be required for heterocyst differentiation, since hetF mutants accumulating HetR are unable to differentiate (Risser and Callahan, 2008). 
414 Similarly, strains bearing several alleles of hetR that result in reduced turnover are also unable to differentiate (Risser and Callahan, 2007). Specific NsiR1 expression in developing heterocysts would reduce HetF accumulation resulting in increased amount of HetR in these cells, favoring expression of HetRdependent transcripts and progression of differentiation (Fig. 5). Finally, despite the small difference in sequence between NsiR1.1 and NsiR1.4 (Supporting Information Fig. S3), strains OE_NsiR1.1 and OE_NsiR1.4 had slightly different phenotypes. They had some growth difference in solid media with different nitrogen sources (Fig. 4A). In fact, the observation that the effect of OE_NsiR1.4 is more important than OE_NsiR1.1, while NsiR1.4 is less efficient in repressing hetF expression, suggests that NsiR1.1 and NsiR1.4 might have additional non-overlapping targets related to heterocyst diferentiation. On the other hand, the observation that the strain OE_as_NsiR1, depleted of NsiR1, showed delayed expression of nifH transcripts encoding nitrogenase indicates NsiR1 influences the overall timing of heterocyst maturation.

Further study of additional potential targets for NsiR1, and the characterization of additional heterocyst-specific non-coding RNAs (Brenes-Álvarez et al., 2019) could reveal the importance of these type of molecules in the regulation of this developmental process.

Materials and Methods

Strains and growth conditions 
438 For Northern blot and primer extension analysis, cultures of wild-type, hetR

439

440

441

442

443

444

445

446

447

448

449

450

451

452

453

454

455

456

457

458

459

460

461

462 mutant 216 (Buikema and Haselkorn, 1991) and different derivative strains of Nostoc sp. PCC 7120 (Supporting Information Table S1) were bubbled with an air $/ \mathrm{CO}_{2}$ mixture $(1 \% \mathrm{v} / \mathrm{v})$ and grown photoautotrophically at $30^{\circ} \mathrm{C}$ in $\mathrm{BG} 11$ medium (Rippka et al., 1979) containing ferric citrate instead of ammonium ferric citrate, lacking $\mathrm{NaNO}_{3}$ but containing $6 \mathrm{mM} \mathrm{NH}_{4} \mathrm{Cl}, 10 \mathrm{mM} \mathrm{NaHCO}_{3}$, and $12 \mathrm{mM} N$-tris (hydroxymethyl) methyl-2-aminoethanesulfonic acid-NaOH buffer $(\mathrm{pH} 7.5)\left(\mathrm{BG} 11_{0} \mathrm{C}+6 \mathrm{mM} \mathrm{NH}_{4} \mathrm{Cl}\right)$. Nitrogen deficiency was induced by filtering, washing and resuspending cells in nitrogen-free-medium with $10 \mathrm{mM} \mathrm{NaHCO}_{3}$ (BG110C). Chlorophyll concentration of the cultures was measured as described (MacKinney, 1941). For Western blot analysis, cultures of different derivative strains of Nostoc sp. PCC 7120 (Supporting Information Table S1) were grown in flasks at $30^{\circ} \mathrm{C}$ in liquid BG11 medium for one week. Nitrogen deficiency was induced as described above. OE_C, OE_NsiR1.1, OE_NsiR1.4 and OE_as_NsiR1 strains were grown in the presence of streptomycin (Sm) and spectinomycin (Sp), $2 \mu \mathrm{g} / \mathrm{ml}$ each (liquid medium) or $5 \mu \mathrm{g} / \mathrm{ml}$ each (solid medium). Nostoc strains bearing $\mathrm{Nm}^{\mathrm{R}}$ plasmids (pMBA92, pMBA93 or pMBA94) were grown in the presence of neomycin $(\mathrm{Nm}), 5 \mu \mathrm{g} / \mathrm{ml}$ (liquid medium) or 25 $\mu \mathrm{g} / \mathrm{ml}$ (solid medium). E. coli strains were grown in LB medium, supplemented with appropriate antibiotics (Sambrook and Russell, 2001).

\section{Construction of Nostoc strains with altered levels of NsiR1}

Plasmids and oligonucleotides used in this work are described in Supporting Information, Tables S2 and S3. The sequences of all fragments amplified by 
463 PCR were entirely verified by sequencing. We have used pMBA37 (Olmedo464 Verd et al., 2019) as a backbone for overexpressing different copies of nsiR1 or an antisense to $n s i R 1$. pMBA37 contains the trc promoter and the $T 1$ terminator from E. coli rrnB gene. The trc promoter from pMBA37 was amplified using oligonucleotides 573 and 574 . This product and oligonucleotide 573 were later used for PCRs with oligonucleotides 757,758 or 512 generating fragments in which the trc promoter was fused respectively to $n \operatorname{siR1.1}$ (fragment 1), nsiR1.4 (fragment 2) or as_nsiR1 (fragment 3). After digestion of fragments 1-3 with Clal and Xhol in the sites provided by oligonucleotides $573,757,758$ and 512 , these fragments were cloned into Clal-Xhol digested pMBA37 vector, rendering respectively pMBA77, pMBA78 and pMBA42 (Supporting Information Table S2). pMBA77, pMBA78 and pMBA42 were introduced in Nostoc sp. PCC 7120 wild type by conjugation (Elhai and Wolk, 1988) generating strains OE_NsiR1.1,

OE_NsiR1.4 and OE_as_NsiR1, respectively (Supporting Information Table

Construction of Nostoc strain with 5'UTR of hetF fused to gfp

We have used pMBA91, a derivative of pMBA20 (Olmedo-Verd et al., 2019), as a backbone for the constitutive overexpression from $r n p B$ promoter of a translational fusion to GFP of the 5'UTRs of hetF. As a control, the gfpmut2 gene was cloned with its own 5'UTR . pMBA91 is similar to pMBA20 but with a $\mathrm{Nm}$ resistance gene instead of a $\mathrm{SmSp}$ resistance cassette. The $\mathrm{Nm}$ resistance gene from pRL278 (Black et al., 1993) was amplified using oligonucleotides 892 
pMBA20, rendering pMBA91 (Supporting Information Table S2). The hetF

5'UTR was amplified using as template genomic DNA and oligonucleotides 555

+ 895. gfpmut2 gene was obtained by PCR using as template pSAM270 (Brenes-Álvarez et al., 2019) and oligonucleotides 896 + 894. A PCR product with a translational fusion between the hetF 5'UTR plus sequences encoding the first 20 amino acids of HetF and gfpmut2 was amplified using as templates the two above-described PCR fragments and oligonucleotides $555+894$. The PCR product was digested with Nsil and Xhol and cloned in Nsil-Xhol digested pMBA91, rendering pMBA93. The 5'UTR of gfpmut2 was used as control. The gfpmut2 gene plus its 5'UTR were amplified using as template pSAM270 (Brenes-Álvarez et al., 2019) and oligonucleotides $894+899$. The PCR product was digested with Nsil and Xhol and cloned in Nsil-Xhol digested pMBA91, rendering pMBA92 (Supporting Information Table S2). pMBA92 and pMBA93 were introduced in Nostoc sp. PCC 7120 by conjugation (Elhai and Wolk, 1988). Sequences of inserts in plasmids expressing GFP translational fusions in Nostoc sp. PCC 7120 are shown in Supporting Information Table S4.

In vitro synthesis and labelling of RNA

506

RNA transcripts were generated in vitro with MEGAscript High Yield

Transcription Kit (AM1333, Ambion). The DNA templates for the transcription of hetF 5'UTR, NsiR1.1 and NsiR1.4 were generated by PCR with a primer that includes a T7 promoter sequence upstream the 5'-end of the coded RNA, and a

511 primer matching the 3'-end of the RNA (Supporting Information Table S3). The

512 templates used for these PCR amplifications were genomic DNA (for hetF 
513 5'UTR), or only the overlapping oligonucleotides (for NsiR1.1 and NsiR1.4). The

514 sequences of the RNAs transcribed in vitro are shown in Supporting Information

515 Table S5. Three (hetF) or one (nsiR1) non-encoded guanosines were added at

516 the 5' end of each template for efficient T7 transcription. After in vitro

517 transcription, RNAs were treated with DNase I and purified by phenol,

518 phenol/chloroform and chloroform extractions, ethanol-precipitated at $-20{ }^{\circ} \mathrm{C}$,

519 and washed with $70 \%$ ethanol. 100 pmol of RNAs were labeled at the 5 '-end

520 with $\left[\gamma^{32}\right.$ P]ATP and polynucleotide kinase and purified in a $6 \%$ polyacrylamide

521 gel as described (Álvarez-Escribano et al., 2018).

522

523

In vitro structure probing and footprinting

524

525

For structure probing of NsiR1.1 and NsiR1.4, $0.1 \mathrm{pmol}$ of labeled NsiR1.1 and

NsiR1.4 RNAs in a $7 \mu$ volume were denatured for $1 \mathrm{~min}$ at $95^{\circ} \mathrm{C}$ and chilled

on ice for $5 \mathrm{~min}$. For the footprinting of NsiR1.1 on hetF 5'UTR, $0.1 \mathrm{pmol}$ of labeled hetF 5'UTR RNA was mixed in $7 \mu$ with water (-) or 1 pmol NsiR1.1 (+), denatured for $1 \mathrm{~min}$ at $95{ }^{\circ} \mathrm{C}$ and chilled on ice for $5 \mathrm{~min}$. After denaturing and chilling steps, we added to all samples $1 \mu \mathrm{l}$ of $1 \mathrm{mg} / \mathrm{ml}$ yeast RNA (Ambion AM7118) and $1 \mu$ of $10 x$ structure buffer (Ambion). The samples were incubated further for $15 \mathrm{~min}$ at $37^{\circ} \mathrm{C}$. RNase T1, RNase A and lead(II) acetate treatment were performed as described (Álvarez-Escribano et al., 2018).

Alkaline and RNase T1 G ladders were generated as described (Álvarezgels and bands visualized with a Cyclone Storage Phosphor System. 
540 Total RNA was isolated using hot phenol as described (Mohamed and Jansson, 1989) with modifications (Brenes-Álvarez et al., 2016). Samples for Northern blot hybridization of NsiR1 were separated in 10\% urea-polyacrylamide gels as described (Steglich et al., 2008) (7 $\mu$ g of total RNA). Samples for Northern blot hybridization of longer transcripts (hetR or nifH) were separated in $1 \%$ agarose denaturing formaldehyde gels (10 $\mathrm{\mu g}$ of total RNA) and transferred to Hybond$\mathrm{N}+$ membrane (GE Healthcare) with 20X SSC buffer. Strand-specific ${ }^{32} \mathrm{P}$ labeled probes were prepared with Taq DNA polymerase using a PCR fragment as template and the oligonucleotides specified in Supporting Information Table S3 in a reaction with $\left[\alpha-{ }^{32} \mathrm{P}\right] \mathrm{dCTP}$ and one single oligonucleotide as primer (corresponding to the complementary strand of the sRNA or mRNA to be detected). PCR fragments for NsiR1.1 and NsiR1.4 probes were amplified with oligonucleotides $506+573$ and pMBA77 (NsiR1.1) or pMBA78 (NsiR1.4) as templates. PCR fragments for hetR and nifH probes were amplified from genomic DNA using oligonucleotides hetR8 + hetR9 and $787+788$ respectively. Hybridization to $r n p B$ (Vioque, 1992) or 5 S rRNA probes was used as a loading and transfer control. Primer extension of $5^{\prime}$ ends of hetF was performed as previously described (Muro-Pastor et al., 1999) using $10 \mu \mathrm{g}$ of total RNA and oligonucleotide 105 labeled with [ $\gamma^{32}$ P]ATP. 
562 For HetR Western blot analysis, crude extracts were prepared using glass

563

564

565

566

567

568

569

570

571

572

573

574

575

576

577

578

579

580

581

582

583

584

585

586

beads. Cells from $25 \mathrm{ml}$ cultures of OE_C, OE_NsiR1.1, OE_NsiR1.4 and

OE_as_NsiR1 subjected to $18 \mathrm{~h}$ of nitrogen deficiency were harvested by filtration, washed with TE50/100 buffer (50 mM Tris pH 8, $100 \mathrm{mM}$ EDTA) and resuspended in $500 \mu \mathrm{l}$ of resuspension buffer $(50 \mathrm{mM}$ Tris- $\mathrm{HCl}$ pH 8.0, 2 mM 2mercaptoethanol) containing a protease inhibitor cocktail (Roche). The cellular suspension was mixed with glass beads (SIGMA, $200 \mu \mathrm{m}$ ) in an Eppendorf tube and subjected to 7 cycles of 1 min vortexing plus $1 \mathrm{~min}$ of cooling on ice. Cell extract was separated from cell debris and unbroken cells by centrifugation (3 $\min$ at $3000 \times g$ at $4^{\circ} \mathrm{C}$ ). The soluble fraction was obtained by centrifugation of the crude extract at $16.000 \times \mathrm{g}$ for $30 \mathrm{~min}$ at $4{ }^{\circ} \mathrm{C}$. The protein concentration was determined by the Lowry procedure (Markwell et al., 1978). $40 \mu \mathrm{g}$ of proteins were fractionated on $10 \%$ SDS-PAGE. Antibodies against Nostoc sp. PCC 7120 HetR (Valladares et al., 2016) and E. coli GroEL (Sigma-Aldrich) were used.

Reporter assay for in vivo verification of targets

We used the GFP reporter system described in (Urban and Vogel, 2007) with the superfolder GFP (sfGFP) and plasmid pXG10-SF (Corcoran et al., 2012) for the experimental verification of targets in E.coli. The 5'UTR of hetF from the TSS at position -257 (4273166f) plus 60 nucleotides within its coding region was amplified using oligonucleotides $555+557$. The GTG start codon was changed to ATG using overlapping PCRs and oligonucleotides 555, 556, 557 and 558. The information about TSS was taken from Mitschke et al. (2011). To 
express NsiR1.1 and NsiR1.4 in E. coli, the genes were amplified from genomic DNA using primers 559 (5' phosphorylated) + 561 or 560 (5' phosphorylated) + 562, respectively. These PCR products were digested with Xbal and fused to a plasmid backbone that was amplified from pZE12-luc with primers PLlacOB and PLlacOD and digested with Xbal, rendering pMML3 and pMML4 (Supporting Information Table S2). Different combinations of plasmids were introduced in $E$. coli DH5a and GFP fluorescence measurements were carried out by either flow cytometry or with a microplate reader (Varioskan) using liquid cultures from individual colonies, as previously described (Wright et al., 2013). Plasmid pJV300 (Supporting Information, Table S2) was used as a control. Sequences of inserts from plasmids used in the heterologous reporter system are shown in Supporting Information Table S6.

\section{Fluorescence microscopy}

Fluorescence of Nostoc sp. PCC 7120 filaments carrying plasmid pMBA92, pMBA93 or pMBA94 (Supporting Information Table S2) growing on top of solidified nitrogen-free medium, was analyzed and quantified as described (Muro-Pastor, 2014) using a Leica HCX PLAN-APO 63× 1.4 NA oil immersion objective attached to a Leica TCS SP2 laser-scanning confocal microscope. Samples were excited at $488 \mathrm{~nm}$ by an argon ion laser and the fluorescent emission was monitored by collection across windows of 500-538 nm (GFP) and 630-700 nm (photosynthetic pigment autofluorescence).

Filaments were stained with Alcian blue as described (Olmedo-Verd et al., 2005) and visualized using a Olympus BX60 microscope. The frequency of 
612 heterocysts was calculated as the number of cells stained by Alcian blue per at

613 least five hundred individual cells counted. All results are expressed as the average of two biological replicates \pm SD.

615

616 Computational and statistical methods

617

618 To detect $n s i R 1$ homologs in the upstream region of hetF, the HetF sequence of Nostoc sp. PCC 7120 was used in a blastp search in IMG (Markowitz et al., 2014) against 100 cyanobacterial genomes, those used in Shih et al. (2013), excluding the genomes corresponding to the fast-evolving Prochlorococcus clade. 50 HetF homologs were identified. We then used one of the identical repeats of $n$ siR1 in Nostoc sp. PCC 7120 as input in a blastn search to predict putative nsiR1 homologs in the region $(4 \mathrm{~kb})$ upstream each of the 50 predicted hetF homologs. Sequences of $n s i R 1$ homologs that were not in the upstream region of hetF were taken from (Brenes-Álvarez et al., 2016). All the NsiR1

627 homologs identified are shown aligned in Supporting Information Fig. S6. The

628 Student's t test was used to determine statistical significance. Number of biological samples can be found in the corresponding figure legends. Models of RNA secondary structures were generated with Mfold (Zuker, 2003)

631 implementing restrictions according to the information obtained from in vitro 632 structure probing.

\section{Supporting information:}


636 Appendix S1: Supporting pdf file, including supplementary Figures S1 to S6 and supplementary Tables S1 to S6.

638

Acknowledgements

640 This work was supported by grants BFU2013-48282-C2-1-P from Ministerio de 641 Economía y Competitividad, and BFU2016-74943-C2-1-P from Agencia Estatal 642 de Investigación (AEI), Ministerio de Economía, Industria y Competitividad, both 643 cofinanced by the European Regional Development Fund, to A.M.M-P. M.B.A is 644 the recipient of a predoctoral contract from Ministerio de Educación, Cultura y 645 Deporte, Spain (FPU014/05123 and EST16-00088). We are grateful to 646 Wolfgang R. Hess (Freiburg University, Germany) for valuable comments on 647 the manuscript.

648

\section{Author contributions}

650

M.B-A., A.V., and A.M.M-P. designed research; M.B-A., M.M., A.V., and A.M.M-P. performed research; M.B-A., A.V., and A.M.M-P. analyzed data; and M.B-A., A.V., and A.M.M-P. wrote the paper.

654

\section{Declaration of interest}

656

The authors declare no conflict of interest.

658

659

\section{References}


661 Álvarez-Escribano, I., Vioque, A., and Muro-Pastor, A.M. (2018) NsrR1, a nitrogen stress-repressed SRNA, contributes to the regulation of $n b / A$ in Nostoc sp. PCC 7120. Front Microbio/ 9: 2267.

Black, T.A., Cai, Y., and Wolk, C.P. (1993) Spatial expression and autoregulation of hetR, a gene involved in the control of heterocyst development in Anabaena. Mol Microbiol 9: 77-84.

667 Brenes-Álvarez, M., Vioque, A., and Muro-Pastor, A.M. (2020) The integrity of the cell wall and its remodeling during heterocyst differentiation are regulated by phylogenetically conserved small RNA Yfr1 in Nostoc sp. strain PCC 7120. mBio 1: e02599-02519. Brenes-Álvarez, M., Olmedo-Verd, E., Vioque, A., and Muro-Pastor, A.M. (2016) Identification of conserved and potentially regulatory small RNAs in heterocystous cyanobacteria. Front Microbiol 7: 48. Brenes-Álvarez, M., Mitschke, J., Olmedo-Verd, E., Georg, J., Hess, W.R., Vioque, A., and Muro-Pastor, A.M. (2019) Elements of the heterocyst-specific transcriptome unravelled by co-expression analysis in Nostoc sp. PCC 7120. Environ Microbiol 21: 2544-2558.

Buikema, W.J., and Haselkorn, R. (1991) Characterization of a gene controlling heterocyst differentiation in the cyanobacterium Anabaena 7120. Genes Dev 5: 321-330.

681 Callahan, S.M., and Buikema, W.J. (2001) The role of HetN in maintenance of 682 the heterocyst pattern in Anabaena sp. PCC 7120. Mol Microbiol 40: 941-950.

683 Corcoran, C.P., Podkaminski, D., Papenfort, K., Urban, J.H., Hinton, J.C., and 684 Vogel, J. (2012) Superfolder GFP reporters validate diverse new mRNA targets of the classic porin regulator, MicF RNA. Mol Microbiol 84: 428-445. Elhai, J., and Wolk, C.P. (1988) Conjugal transfer of DNA to cyanobacteria. Methods Enzymol 167: 747-754.

688 Elhai, J., and Khudyakov, I. (2018) Ancient association of cyanobacterial 689 multicellularity with the regulator HetR and an RGSGR pentapeptide-containing 690 protein (PatX). Molecular Microbiology 110: 931-954.

691 Flores, E., and Herrero, A. (2010) Compartmentalized function through cell 692 differentiation in filamentous cyanobacteria. Nat Rev Microbiol 8: 39-50. 
693 Flores, E., Picossi, S., Valladares, A., and Herrero, A. (2019) Transcriptional

694

695

696

697

698

699

700

701

702

703

704

705

706

707

708

709

710

711

712

713

714

715

716

717

718

719

720

721

722

723

724

725

726 regulation of development in heterocyst-forming cyanobacteria. Biochim Biophys Acta Gene Regul Mech 1862: 673-684.

Herrero, A., Muro-Pastor, A.M., and Flores, E. (2001) Nitrogen control in cyanobacteria. J Bacteriol 183: 411-425.

Herrero, A., Stavans, J., and Flores, E. (2016) The multicellular nature of filamentous heterocyst-forming cyanobacteria. FEMS Microbiol Rev 40: 831-854. Huang, X., Dong, Y., and Zhao, J. (2004) HetR homodimer is a DNA-binding protein required for heterocyst differentiation, and the DNA-binding activity is inhibited by PatS. Proc Natl Acad Sci USA 101: 4848-4853.

Ionescu, D., Voß, B., Oren, A., Hess, W.R., and Muro-Pastor, A.M. (2010) Heterocyst-specific transcription of NsiR1, a non-coding RNA encoded in a tandem array of direct repeats in cyanobacteria. J Mol Biol 398: 177-188.

Jagodnik, J., Brosse, A., Le Lam, T.N., Chiaruttini, C., and Guillier, M. (2017) Mechanistic study of base-pairing small regulatory RNAs in bacteria. Methods 117: $67-76$.

Kopf, M., and Hess, W.R. (2015) Regulatory RNAs in photosynthetic cyanobacteria. FEMS Microbiol Rev 39: 301-315.

MacKinney, G. (1941) Absorption of light by chlorophyll solutions. J Biol Chem 140: 315-322.

Marchais, A., Duperrier, S., Durand, S., Gautheret, D., and Stragier, P. (2011) CsfG, a sporulation-specific, small non-coding RNA highly conserved in endospore formers. RNA Biol 8: 358-364.

Markowitz, V.M., Chen, I.M., Palaniappan, K., Chu, K., Szeto, E., Pillay, M. et al. (2014) IMG 4 version of the integrated microbial genomes comparative analysis system. Nucleic Acids Res 42: D560-D567.

Markwell, M.A., Haas, S.M., Bieber, L.L., and Tolbert, N.E. (1978) A modification of the Lowry procedure to simplify protein determination in membrane and lipoprotein samples. Anal Biochem 87: 206-2010.

Mars, R.A., Nicolas, P., Denham, E.L., and van Dijl, J.M. (2016) Regulatory RNAs in Bacillus subtilis: a Gram-positive perspective on bacterial RNA-mediated regulation of gene expression. Microbiol Mol Biol Rev 80: 1029-1057.

Mitschke, J., Vioque, A., Haas, F., Hess, W.R., and Muro-Pastor, A.M. (2011) Dynamics of transcriptional start site selection during nitrogen stress-induced cell 
727 differentiation in Anabaena sp. PCC 7120. Proc Natl Acad Sci USA 108: 20130-

$728 \quad 20135$.

729 Mohamed, A., and Jansson, C. (1989) Influence of light on accumulation of 730 photosynthesis-specific transcripts in the cyanobacterium Synechocystis 6803.

731 Plant Mol Biol 13: 693-700.

732 Muro-Pastor, A.M. (2014) The heterocyst-specific NsiR1 small RNA is an early 733 marker of cell differentiation in cyanobacterial filaments. MBio 5: e01079-01014. 734 Muro-Pastor, A.M., and Hess, W.R. (2012) Heterocyst differentiation: from single 735 mutants to global approaches. Trends Microbiol 20: 548-557.

736 Muro-Pastor, A.M., and Hess, W.R. (2020) Regulatory RNA at the crossroads of 737 carbon and nitrogen metabolism in photosynthetic cyanobacteria. Biochim 738 Biophys Acta Gene Regul Mech 1863: 194477.

739 Muro-Pastor, A.M., Valladares, A., Flores, E., and Herrero, A. (1999) The hetC 740 gene is a direct target of the NtcA transcriptional regulator in cyanobacterial 741 heterocyst development. J Bacteriol 181: 6664-6669.

742 Muro-Pastor, A.M., Valladares, A., Flores, E., and Herrero, A. (2002) Mutual 743 dependence of the expression of the cell differentiation regulatory protein HetR 744 and the global nitrogen regulator NtcA during heterocyst development. Mol Microbiol 44: 1377-1385.

746 Olmedo-Verd, E., Flores, E., Herrero, A., and Muro-Pastor, A.M. (2005) HetR747 dependent and -independent expression of heterocyst-related genes in an 748 Anabaena strain overproducing the NtcA transcription factor. $J$ Bacteriol 187: 749 1985-1991.

750 Olmedo-Verd, E., Muro-Pastor, A.M., Flores, E., and Herrero, A. (2006) Localized 751 induction of the $n t c A$ regulatory gene in developing heterocysts of Anabaena sp. 752 strain PCC 7120. J Bacteriol 188: 6694-6699.

753 Olmedo-Verd, E., Brenes-Álvarez, M., Vioque, A., and Muro-Pastor, A.M. (2019) 754 A heterocyst-specific antisense RNA contributes to metabolic reprogramming in 755 Nostoc sp. PCC 7120. Plant Cell Physiol 60: 1646-1655.

756 Rajagopalan, R., and Callahan, S.M. (2010) Temporal and spatial regulation of 757 the four transcription start sites of hetR from Anabaena sp. strain PCC 7120. J 758 Bacteriol 192: 1088-1096. 
759 Rippka, R., Deruelles, J., Waterbury, J.B., Herdman, M., and Stanier, R.Y. (1979)

760 Generic assignments, strain histories and properties of pure cultures of cyanobacteria. Microbiology 111: 1-61.

762 Risser, D.D., and Callahan, S.M. (2007) Mutagenesis of hetR reveals amino acids necessary for HetR function in the heterocystous cyanobacterium Anabaena sp. strain PCC 7120. J Bacteriol 189: 2460-2467.

765 Risser, D.D., and Callahan, S.M. (2008) HetF and PatA control levels of HetR in 766 Anabaena sp. strain PCC 7120. J Bacteriol 190: 7645-7654.

767 Risser, D.D., and Callahan, S.M. (2009) Genetic and cytological evidence that heterocyst patterning is regulated by inhibitor gradients that promote activator decay. Proc Natl Acad Sci USA 106: 19884-19888. Sambrook, J., and Russell, D.W. (2001) Molecular cloning : A laboratory manual. New York: Cold Spring Harbor Laboratory.

772 Schmalisch, M., Maiques, E., Nikolov, L., Camp, A.H., Chevreux, B., Muffler, A. et al. (2010) Small genes under sporulation control in the Bacillus subtilis genome. J Bacteriol 192: 5402-5412.

Shih, P.M., Wu, D., Latifi, A., Axen, S.D., Fewer, D.P., Talla, E. et al. (2013) Improving the coverage of the cyanobacterial phylum using diversity-driven genome sequencing. Proc Natl Acad Sci USA 110: 1053-1058.

Silvaggi, J.M., Perkins, J.B., and Losick, R. (2006) Genes for small, noncoding RNAs under sporulation control in Bacillus subtilis. J Bacteriol 188: 532-541. Steglich, C., Futschik, M.E., Lindell, D., Voss, B., Chisholm, S.W., and Hess, W.R. (2008) The challenge of regulation in a minimal photoautotroph: non-coding RNAs in Prochlorococcus. PLoS Genet 4: e1000173. Urban, J.H., and Vogel, J. (2007) Translational control and target recognition by Escherichia coli small RNAs in vivo. Nucleic Acids Res 35: 1018-1037. Valladares, A., Flores, E., and Herrero, A. (2016) The heterocyst differentiation transcriptional regulator HetR of the filamentous cyanobacterium Anabaena forms tetramers and can be regulated by phosphorylation. Mol Microbio/ 99: 808819.

Vioque, A. (1992) Analysis of the gene encoding the RNA subunit of ribonuclease P from cyanobacteria. Nucleic Acids Res 20: 6331-6337. who they are, what they do, and how they do it. Adv Genet 90: 133-208. 
793 Wong, F.C., and Meeks, J.C. (2001) The hetF gene product is essential to 794 heterocyst differentiation and affects HetR function in the cyanobacterium Nostoc 795 punctiforme. J Bacteriol 183: 2654-2661.

796 Wright, P.R., Richter, A.S., Papenfort, K., Mann, M., Vogel, J., Hess, W.R. et al. 797 (2013) Comparative genomics boosts target prediction for bacterial small RNAs. 798 Proc Natl Acad Sci USA 110: E3487-E3496.

799 Yoon, H.S., and Golden, J.W. (1998) Heterocyst pattern formation controlled by 800 a diffusible peptide. Science 282: 935-938.

801 Zhou, R., Wei, X., Jiang, N., Li, H., Dong, Y., Hsi, K.L., and Zhao, J. (1998) 802 Evidence that HetR protein is an unusual serine-type protease. Proc Natl Acad 803 Sci USA 95: 4959-4963.

804 Zuker, M. (2003) Mfold web server for nucleic acid folding and hybridization 805 prediction. Nucleic Acids Res 31: 3406-3415.

806

807 
Fig. 1. NsiR1 interacts with hetF 5' UTR.

A. Schematic representation of the genomic region encoding NsiR1 in Nostoc sp. PCC 7120 and constructs in plasmids pMBA92 and pMBA93. Bent arrows indicate the transcriptional start sites. Identical repeats of $n \operatorname{siR} 1$ (3-9, e.g. $n s i R 1.4)$ are shown as red arrows. Repeats of $n s i R 1$ with some sequence divergence $(1,2,10,11$ and 12) are shown in light blue except repeat 1 (nsiR1.1), antisense to hetF, that is shown in green.

B. Top, confocal fluorescence images (green channel, red channel and bright field) of filaments from Nostoc strains bearing plasmids pMBA92 (control, gfp gene expressed from the $r n p B$ promoter) or pMBA93 (translational fusion, expressed from the $r n p B$ promoter, of the hetF 5' UTR to the gfp gene) growing on top of nitrogen-free medium. Bottom, quantification of the signals for the

822 green (GFP) and red (autofluorescence) channels. The loss of autofluorescence

823 from photosynthetic pigments is a characteristic feature of heterocyst

824 differentiation. Heterocysts are indicated with numbers. C. GFP fluorescence of $E$. coli cultures bearing different combinations of a plasmid expressing 5' UTR hetF::sfgfp with plasmids expressing NsiR1.1,

827 NsiR1.4 or an unrelated control RNA (pJV300, see Materials and Methods for 828 details). The data are represented as mean and standard deviation of results 829 from cultures corresponding to four independent colonies after normalization for 830 cell density and subtraction of background fluorescence in cells bearing pXG0.

831 T-test P-value $<0.05^{*} ;<0.01^{* *}$. 
D. FACS-based reporter assays. The plot shows the distribution of fluorescence

833 in 10,000 E. coli cells bearing plasmids expressing 5' UTR hetF:sfgfp and the 834 unrelated control RNA (black), NsiR1.1 (green) or NsiR1.4 (red). The inset shows the average fluorescence of each sample.

Fig. 2. In vitro footprinting assay of the interaction between NsiR1.1 and the 837 hetF 5' UTR.

838 A. 5'-labeled hetF 5' UTR RNA was treated with RNase T1, RNase A, or lead(II) acetate $\left(\mathrm{Pb}^{+2}\right)$ in the absence $(-)$ or presence $(+)$ of NsiR1.1. Samples were loaded on two gels that were run to different lengths. Vertical bars indicate the NsiR1.1 binding site (red), a region with increased sensitivity to the probes in the presence of NsR1.1 (green) and the region including the start codon (orange). Nucleotides with differential sensitivity to RNase T1 or RNase A in the presence or absence of NsiR1.1 are indicated with colored triangles. Nucleotide positions of hetF 5' UTR are shown on the left. C, untreated control; $\mathrm{OH}$, alkaline ladder; T1s, RNase T1 sequencing ladder.

B, C. Models of the secondary structure of hetF 5' UTR from Nostoc sp. PCC 7120 without (B) or with bound NsiR1.1 (C). Nucleotides corresponding to the areas highlighted by vertical bars in the gels are colored in the same way. Nucleotides with differential sensitivity to RNase T1 or RNase A outside the NsiR1 binding site are indicated with colored triangles. Start codon is in purple with a purple frame. The three non-encoded guanosines added at the 5 ' end of hetF 5'UTR for efficient transcription are shown in lowercase.

Fig. 3. Nostoc sp. PCC 7120 strains with altered levels of NsiR1.

A. Scheme of DNA fragments cloned in the plasmids used to generate the different strains. Transcription start site (bent arrows), Rho-independent 
terminator of NsiR1 (small stem loop), T1 terminator (large stem loop), trc promoter, strand specific probes for NsiR1.1 (green line) and for NsiR1.4 (red line) are indicated.

B. Northern blots using RNA extracted from the different strains at different times after nitrogen removal hybridized with probes for NsiR1.1 (top), NsiR1.4 (middle) and 5S rRNA (bottom) as a loading control. Endogenous NsiR1 (black triangles) and the three RNA species constitutively expressed from the trc promoter, NsiR1.1 (green triangle), NsiR1.4 (red triangle) and as_NsiR1 (grey triangles), are indicated. Black bars indicate transcripts whose transcription did not stop at the Rho-independent terminators of $n s i R 1$.

C. Primer extension analysis of hetF transcripts using RNA isolated from the mutant strains at different time points after combined nitrogen removal. The position of the TSS of hetF (4273166f) is indicated with an asterisk. The position corresponding to the TSS of $n \operatorname{siR} 1.1(4273242 r)$ is indicated with a black triangle. Additional stops provoked by constitutive overexpression of NsiR1.1 (green triangle) and NsiR1.4 (red triangle) from the trc promoter are indicated. On the right, a sequencing reaction performed with the same oligonucleotide used for primer extension.

D. Accumulation of HetR in the mutant strains $18 \mathrm{~h}$ after combined nitrogen removal. Western blots were carried out with proteins extracted from three

877 independent cultures of each strain using antibodies against HetR or GroEL 878 (used as loading control).

879 E. Quantification of Western blot shown in (D). The data are presented as the 880 mean \pm standard deviation of the HetR signal normalized to the GroEL signal in 881 the three independent clones of each strain shown in (D). The mean of HetR 
accumulation of the OE_C strain in each gel is used as $100 \% .{ }^{* *} T$-test P-value

Fig. 4. Phenotype of OE_C, OE_NsiR1.1, OE_NsiR1.4 and OE_as_NsiR1 strains.

A. Growth of solid media. Cells from liquid cultures of the indicated strains grown in the presence of nitrate were collected and resuspended in BG110 at an $\mathrm{OD}_{750}=0.17$. Five-fold serial dilutions were prepared and $10 \mu \mathrm{l}$ of each dilution plated on $\mathrm{BG} 11_{0}$ plates containing ammonium $\left(\mathrm{NH}_{4}{ }^{+}\right)$, nitrate $\left(\mathrm{NO}_{3}{ }^{-}\right)$or lacking combined nitrogen $\left(\mathrm{N}_{2}\right)$. Pictures were taken after 10 days of incubation at $30^{\circ} \mathrm{C}$.

B. Bright field images of filaments of OE_C, OE_NsiR1.1, OE_NsiR1.4 and OE_as_NsiR1 strains growing in liquid media containing ammonium $\left(\mathrm{NH}_{4}{ }^{+}\right)$or growing in liquid media without combined nitrogen $24 \mathrm{~h}$ after nitrogen removal $\left(\mathrm{N}_{2}\right)$. Filaments with enlarged irregular cells are pointed by black arrows. Heterocysts were stained with Alcian Blue. Scale bars, $25 \mu \mathrm{m}$. OE_as_NsiR1. The data are presented as the mean and standard deviation of two cultures from two independent colonies. T-test P-value $<0.05^{*} ;<0.01^{* *}$. D. Northern blots with RNA isolated from the indicated strains at different time points (indicated in hours) after nitrogen removal and hybridized with probes for

902 nifH (upper panels) and $r n p B$ (bottom panels) as a loading control. Sizes are 903 indicated on the left in $\mathrm{kb}$.

904 Fig. 5. Model of the function of NsiR1 in the regulation of heterocyst 905 differentiation. Positive regulation is indicated by a blue arrow. Negative post 906 transcriptional regulation by NsiR1 is indicated by a red line with a blunt end. 
907 Different sizes indicate the relative amount of HetF and HetR in vegetative cells 908 versus heterocysts.

909 
Figure 1
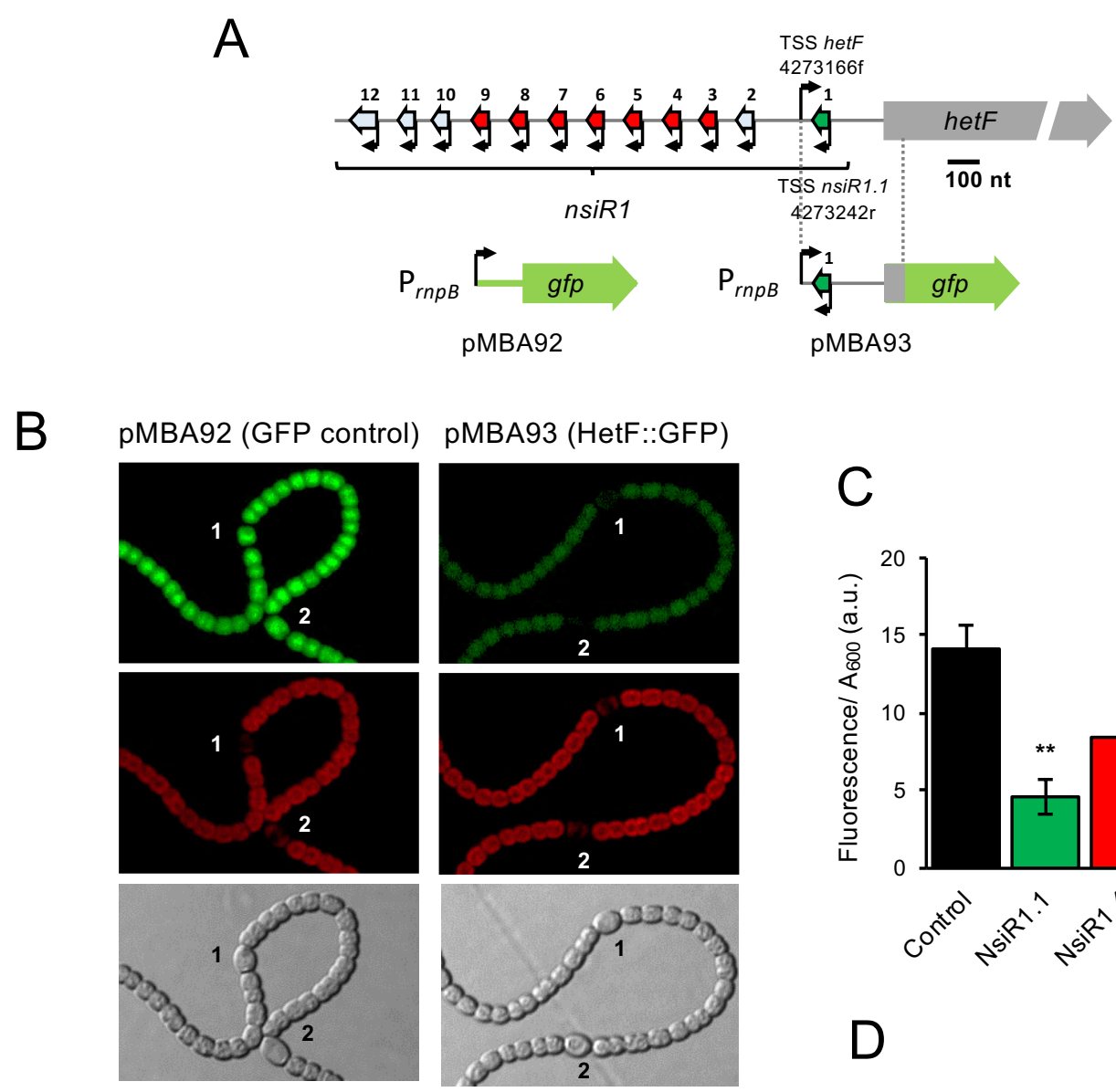

C
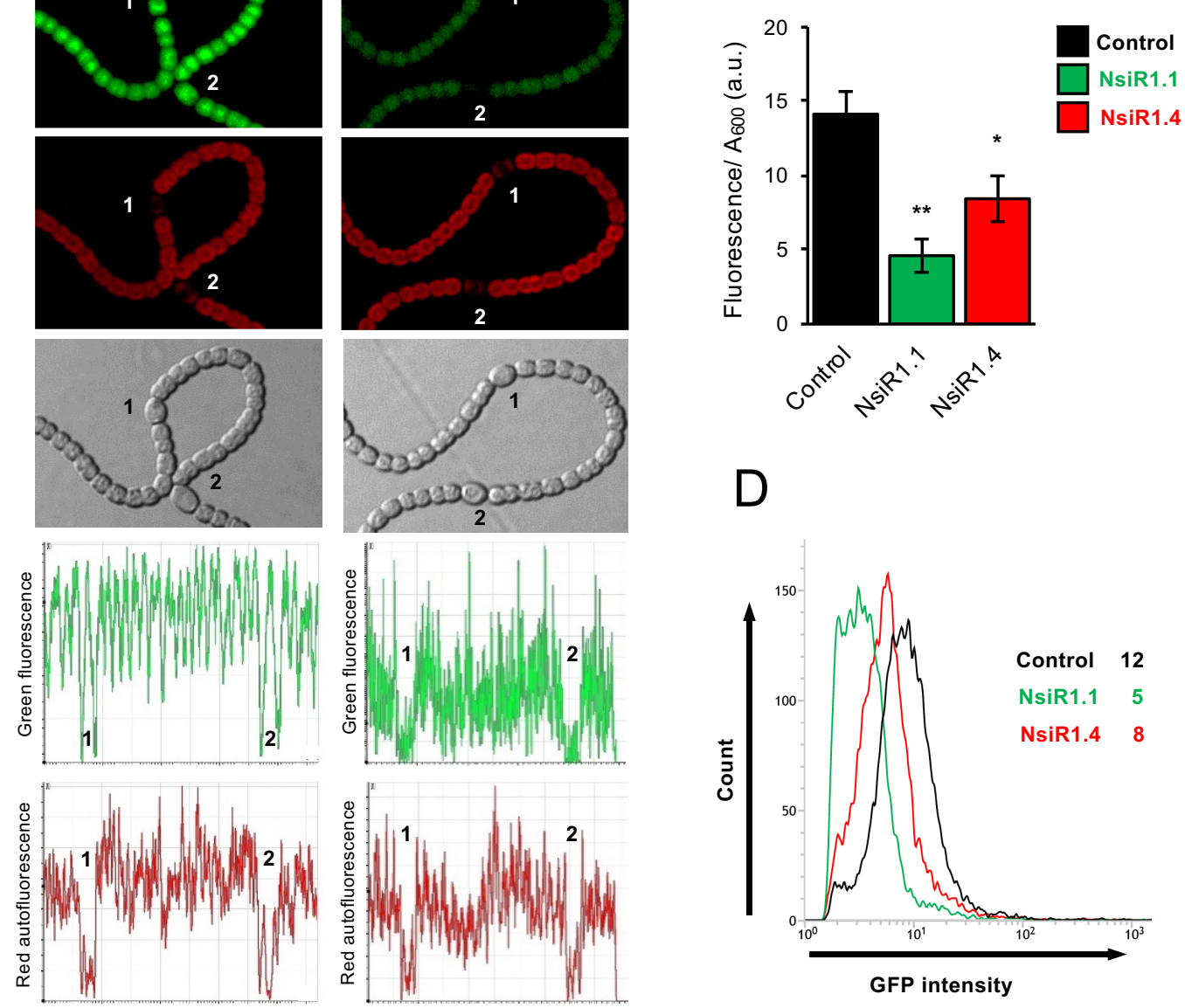


\section{Figure 2}

A

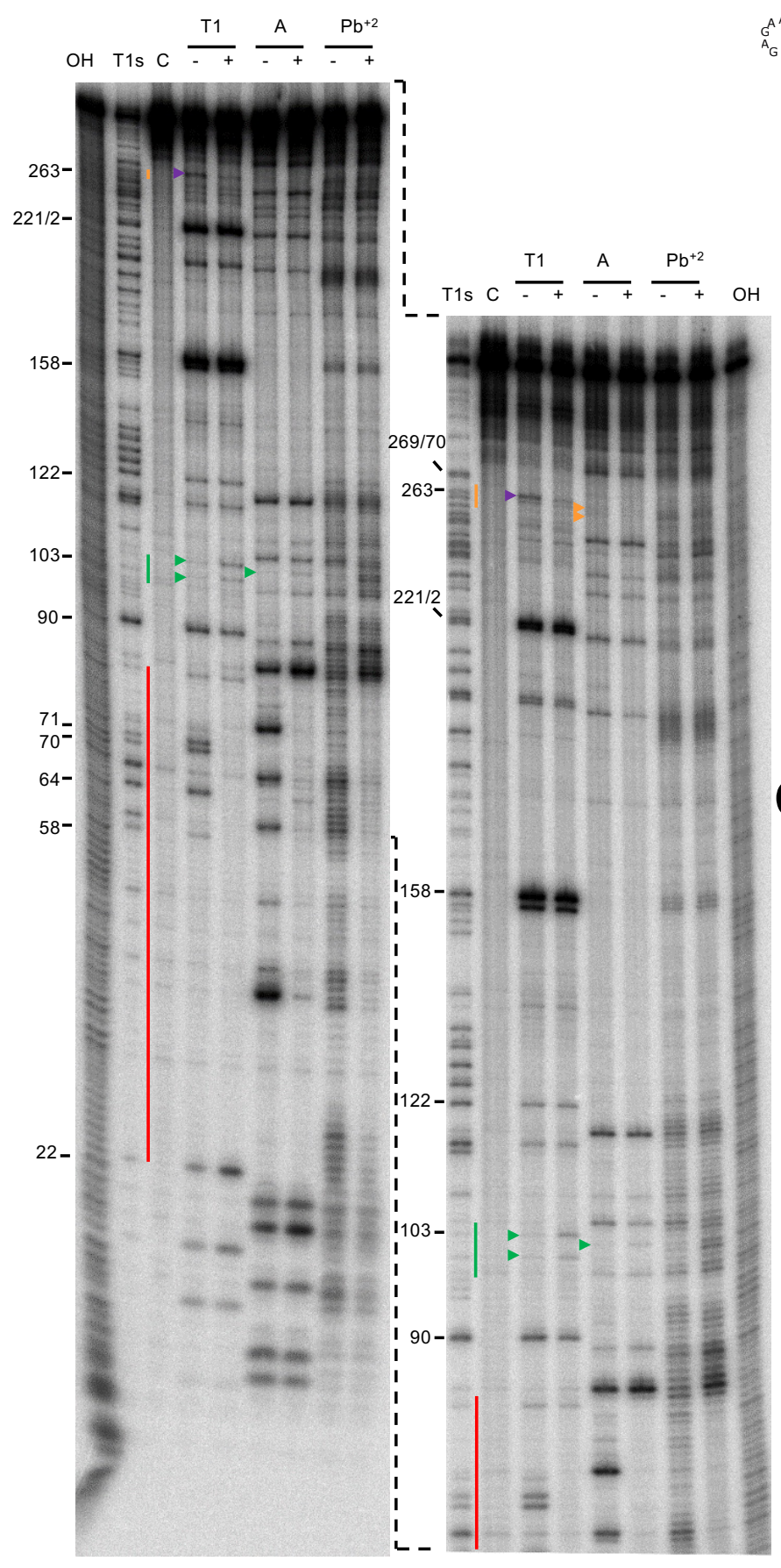

B
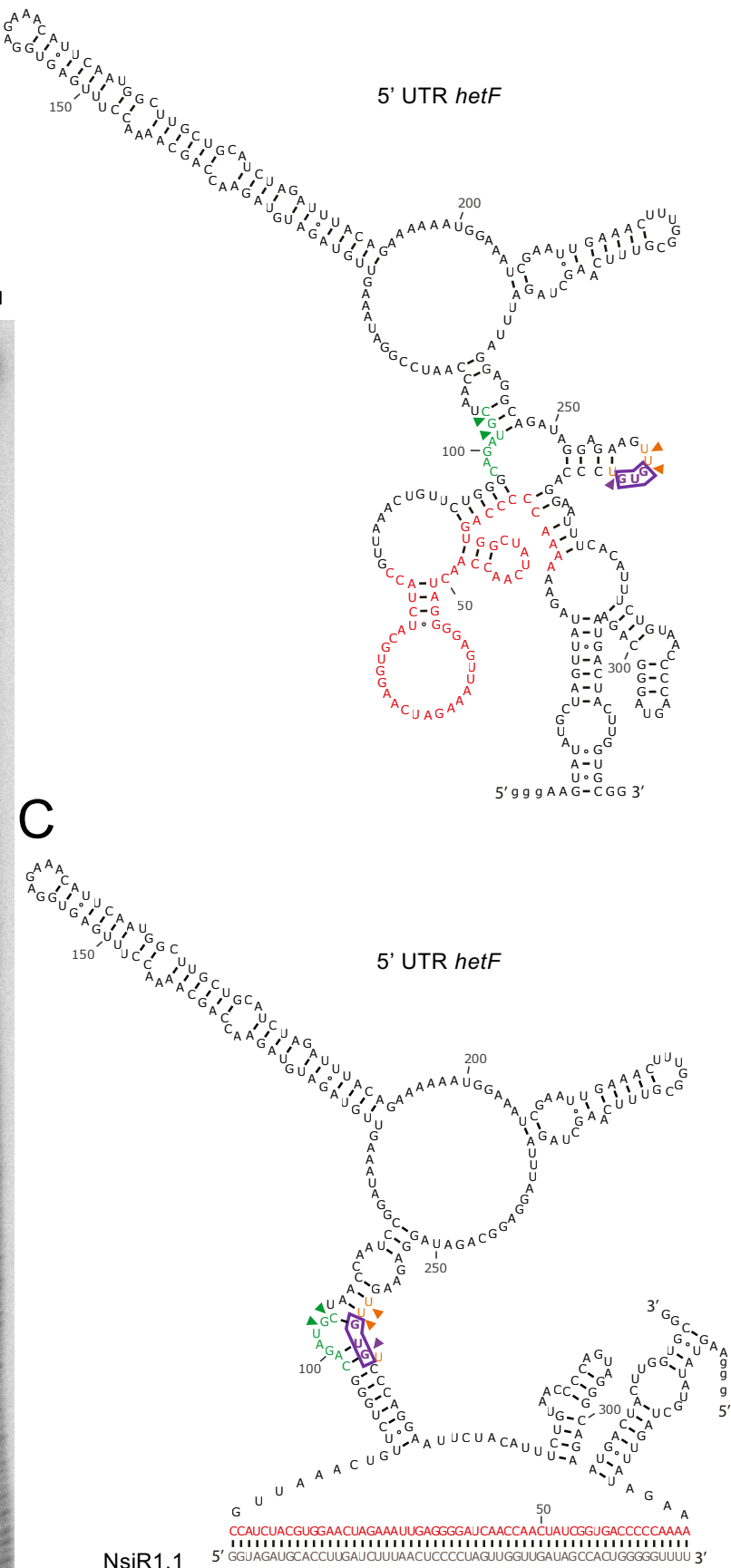


\section{Figure 3}

A

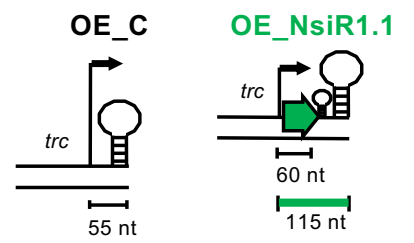

C

OE_NsiR1.4 OE_as_NsiR1

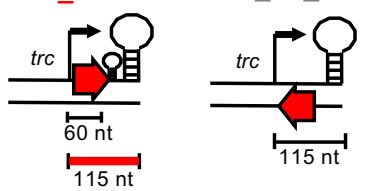

OE_C: blank plasmid control

OE_NsiR1.1: NsiR1.1 overexpression

OE_NsiR1.4: NsiR1.4 overexpression OE_as_NsiR1: NsiR1 depletion

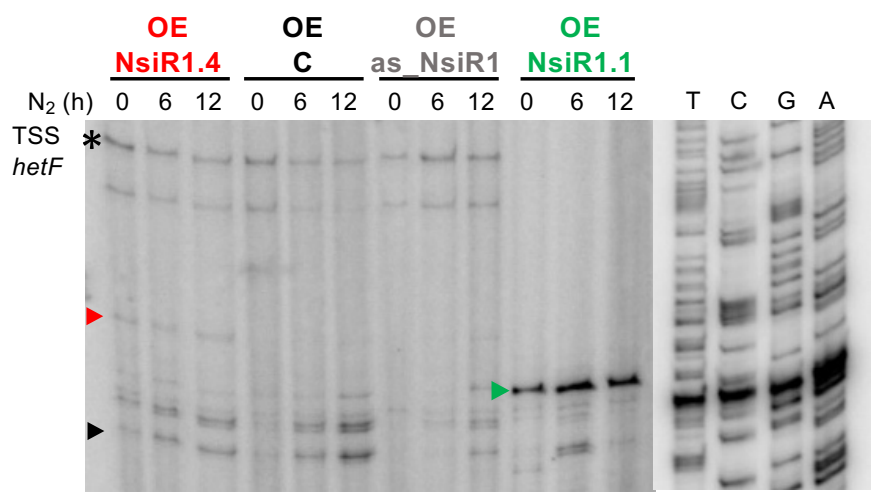

B
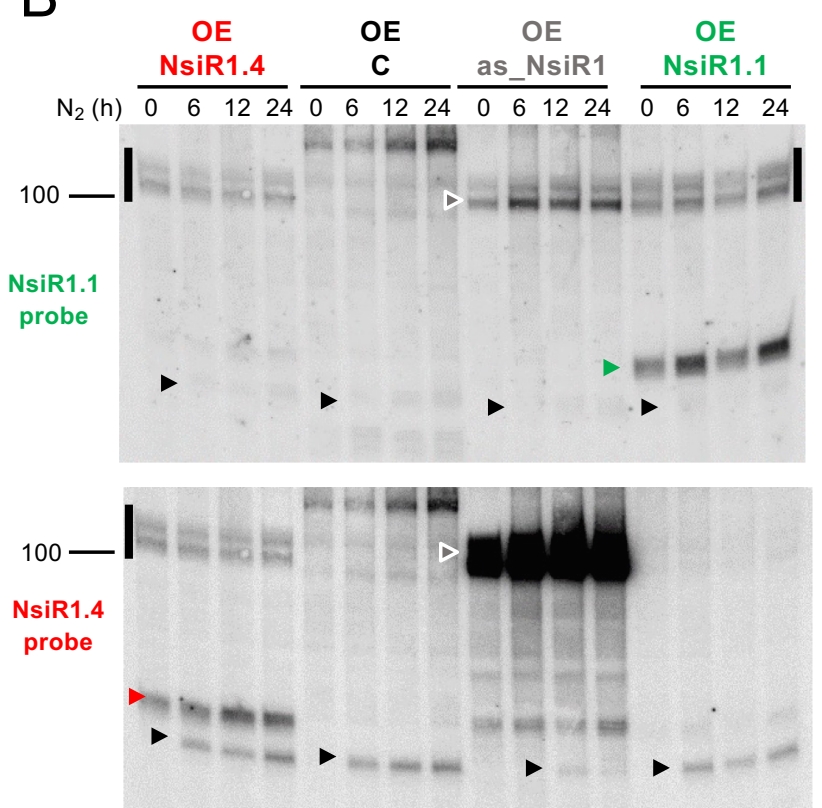

D

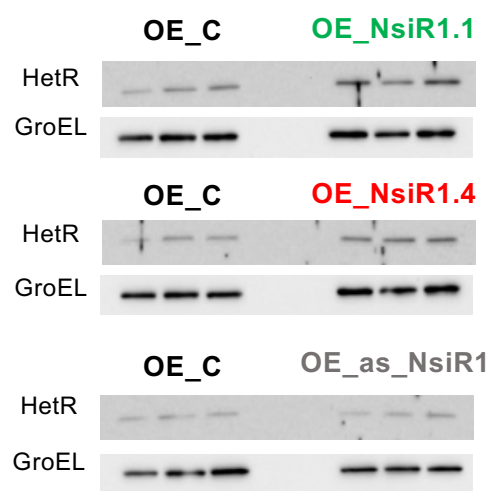

E

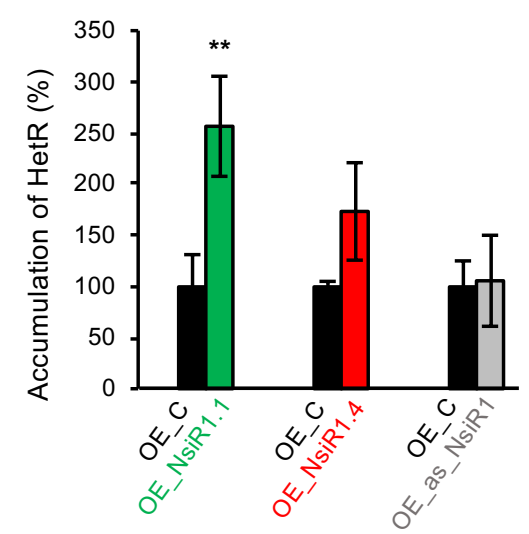

5S RNA 


\section{Figure 4}

A
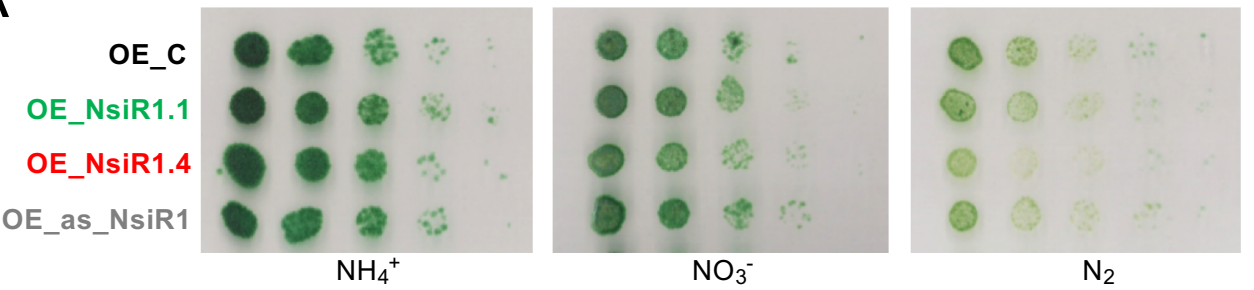

B

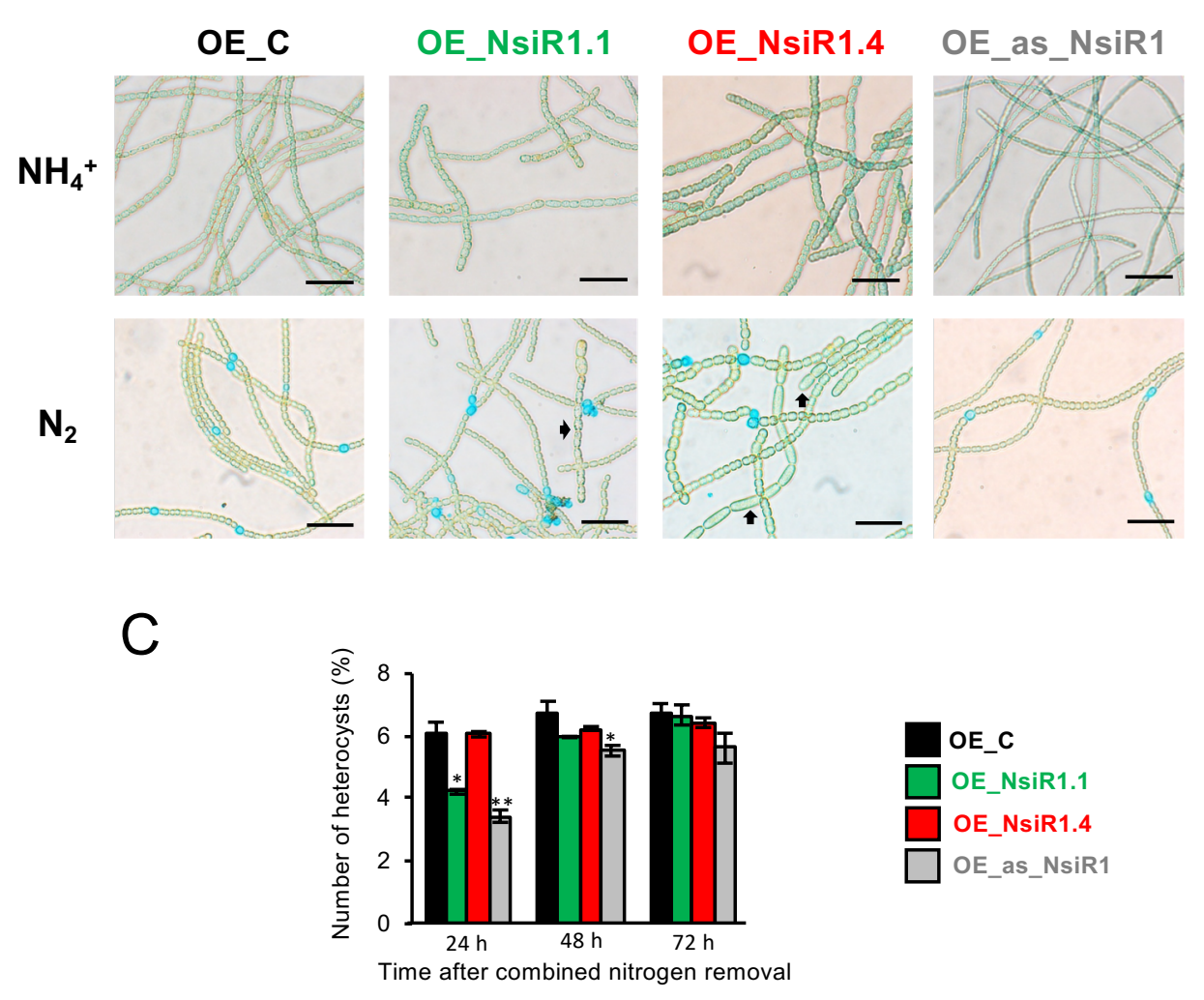

D

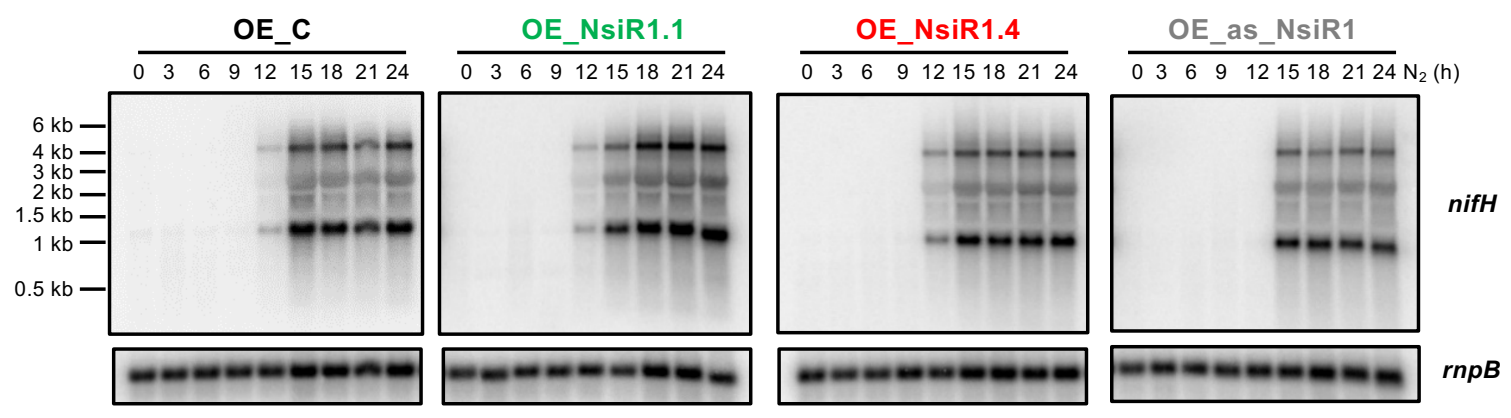


Figure 5

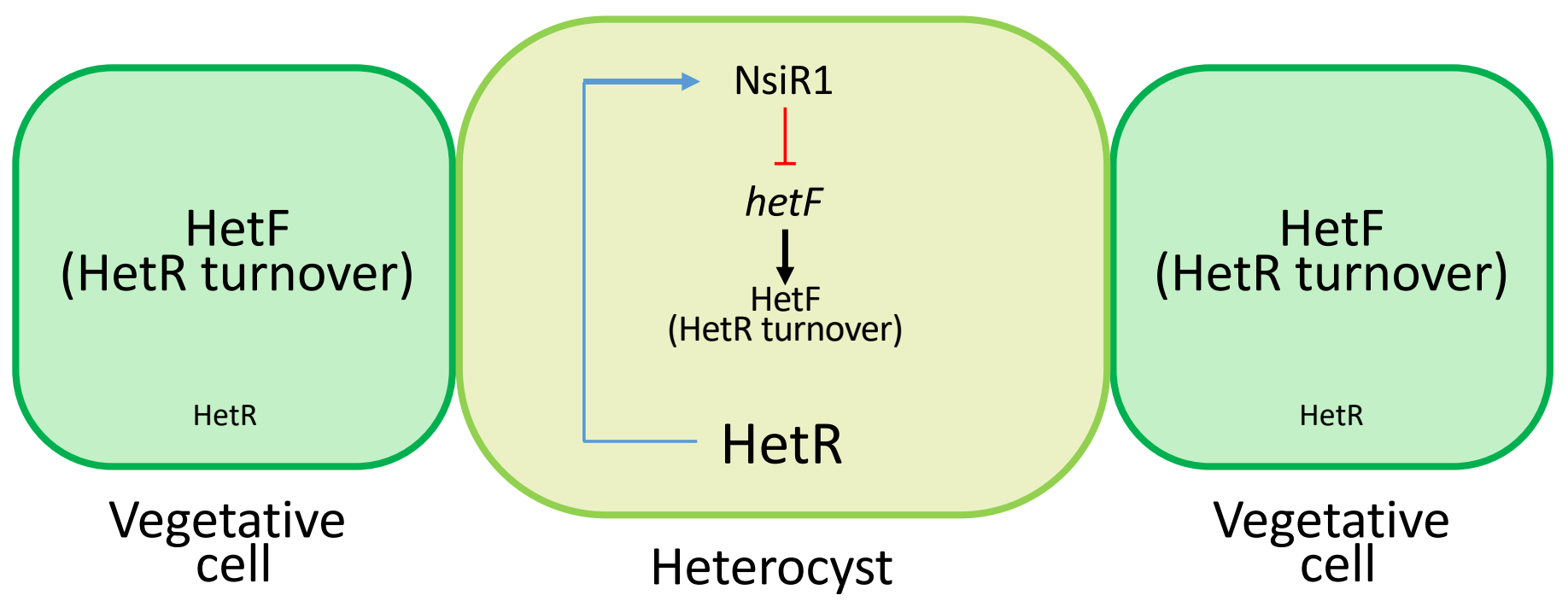




\section{Supplementary Information for}

NsiR1, a small RNA with multiple copies, modulates heterocyst differentiation in Nostoc sp. PCC 7120

Authors: Manuel Brenes-Álvarez, Marina Minguet ${ }^{a}$, Agustín Vioque $^{1}$, and Alicia M. Muro-Pastor

Authors affiliation: Instituto de Bioquímica Vegetal y Fotosíntesis, Consejo Superior de Investigaciones Científicas and Universidad de Sevilla, Sevilla, Spain.

aPresent address: Centro de Biología Molecular Severo Ochoa, CSIC-UAM, Nicolás Cabrera 1, 28049, Madrid, Spain.

${ }^{1}$ Corresponding Author: Agustín Vioque, Instituto de Bioquímica Vegetal y Fotosíntesis, Américo Vespucio 49, 41092, Sevilla, Spain

Phone: +34-954489519

Email: vioque@us.es

This PDF file includes:

Figures S1 to S6

Tables S1 to S6

Supplementary references 


\section{Supplementary Figures}

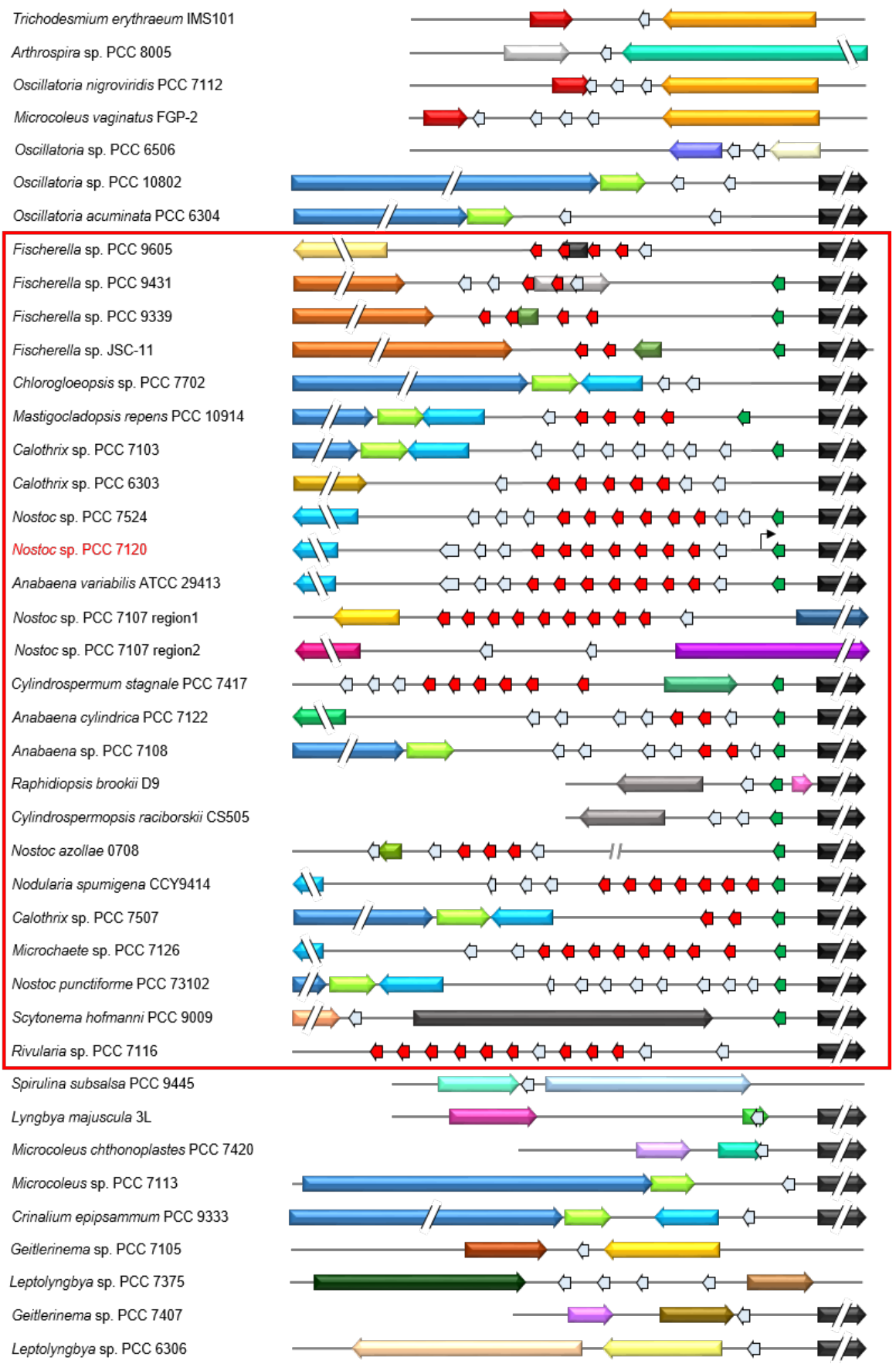

Fig. S1. Genomic regions containing nsiR1 repeats in $\mathbf{4 0}$ cyanobacterial genomes. $n \operatorname{siR} 1$ repeats are indicated by small arrows with solid colors (red for identical repeats in each genome, light blue for repeats with sequence divergence and green for repeats that might be antisense to hetF 5'-UTR). Genes are indicated by arrows with faded colors. Homologous genes are shown in the same color. hetF homologs are shown by dark grey arrows on the right side of the regions. The TSS of hetF in Nostoc sp. PCC 7120 is indicated by a bent arrow. The coherent clade of heterocyst-forming cyanobacteria is highlighted by a red frame. Nostoc sp. PCC 7120 is indicated in red. 


\begin{tabular}{|c|c|c|}
\hline Organism & hetF & $n s i R 1$ \\
\hline Trichodesmium erythraeum IMS101 & + & 1 \\
\hline Arthrospira platensis str. Paraca & + & - \\
\hline Arthrospira platensis NIES-39 & + & - \\
\hline Arthrospira sp. PCC 8005 & + & 1 \\
\hline Arthrospira maxima CS-328 & + & - \\
\hline Oscillatoria nigroviridis PCC 7112 & + & 3 \\
\hline Microcoleus vaginatus FGP-2 & + & 4 \\
\hline Oscillatoria sp. PCC 6506 & + & 2 \\
\hline Oscillatoria formosa PCC 6407 & + & - \\
\hline Oscillatoria sp. PCC 10802 & + & 2 \\
\hline Oscillatoria acuminata PCC 6304 & + & 2 \\
\hline Fischerella sp. 9605 & + & 5 \\
\hline Fischerella sp. PCC 9431 & + & 6 \\
\hline Fischerella sp. PCC 9339 & + & 5 \\
\hline Fischerella sp. PCC JSC-11 & + & 3 \\
\hline Cyanobacterium PCC 7702 & + & 2 \\
\hline Mastigocladopsis repens PCC 10914 & + & 6 \\
\hline Calothrix sp. PCC 7103 & + & 8 \\
\hline Calothrix sp. PCC 6303 & + & 8 \\
\hline Nostoc sp. PCC 7524 & + & 12 \\
\hline Nostoc sp. PCC 7120 & + & 12 \\
\hline Anabaena variabilis ATCC 29413 & + & 12 \\
\hline Nostoc sp. PCC 7107 & + & 13 \\
\hline Cylindrospermum stagnale PCC 7417 & + & 10 \\
\hline Anabaena cylindrica PCC 7122 & + & 8 \\
\hline Anabaena sp. PCC 7108 & + & 8 \\
\hline Raphidiopsis brookii D9 & + & 2 \\
\hline Cylindrospermopsis raciborskii CS-505 & + & 3 \\
\hline Nostoc azollae 0708 & + & 7 \\
\hline Nodularia spumigena CCY 9414 & + & 11 \\
\hline Calothrix sp. PCC 7507 & + & 3 \\
\hline Microchaete sp. PCC 7126 & + & 11 \\
\hline Nostoc punctiforme PCC 73102 & + & 9 \\
\hline Scytonema hofmanni UTEX 2349 & + & 2 \\
\hline Rivularia sp. PCC 7116 & + & 12 \\
\hline Synechocystis sp. PCC 7509 & - & - \\
\hline Gloeocapsa sp. PCC 7428 & - & - \\
\hline Chroococcidiopsis thermalis PCC 7203 & - & - \\
\hline Dactylococcopsis salina PCC 8305 & - & - \\
\hline Halothece sp. PCC 7418 & - & - \\
\hline Spirulina subsalsa PCC 9445 & + & 1 \\
\hline Spirulina major PCC 6313 & + & - \\
\hline Cyanothece sp. CCY0110 & - & - \\
\hline Cyanothece sp. ATCC 51472 & - & - \\
\hline Cyanothece sp. ATCC 51142 & - & - \\
\hline
\end{tabular}

\begin{tabular}{|c|c|c|}
\hline Organism & hetF & nsiR1 \\
\hline Crocosphaera watsonii WH0003 & - & - \\
\hline Crocosphaera watsonii WH8501 & - & - \\
\hline Cyanothece sp. PCC 8802 & - & - \\
\hline Cyanothece sp. PCC 8801 & - & - \\
\hline Synechocystis sp. PCC 6803 & - & - \\
\hline Pleurocapsa sp. PCC 7327 & - & - \\
\hline Microcystis aeruginosa NIES-843 & - & - \\
\hline Cyanothece sp. PCC 7822 & - & - \\
\hline Cyanothece sp. PCC 7424 & - & - \\
\hline Gloeocapsa sp. PCC 73106 & - & - \\
\hline Synechococcus sp. PCC 7002 & - & - \\
\hline Leptolyngbya sp. PCC 7376 & + & - \\
\hline Cyanobacterium stanieri PCC 7202 & - & - \\
\hline Geminocystis herdmanii PCC 6308 & - & - \\
\hline Cyanobacterium aponinum PCC 10605 & - & - \\
\hline Stanieria cyanosphaera PCC 7437 & - & - \\
\hline Pleurocapsa sp. PCC 7319 & - & - \\
\hline Xenococcus sp. PCC 7305 & - & - \\
\hline Lyngbya majuscula $3 \mathrm{~L}$ & + & 1 \\
\hline Microcoleus chthonoplastes PCC 7420 & + & 1 \\
\hline Microcoleus sp. PCC 7113 & + & 1 \\
\hline Crinalium epipsammum PCC 9333 & + & 1 \\
\hline Chamaesiphon minutus PCC 6605 & - & - \\
\hline Geitlerinema sp. PCC 7105 & + & 1 \\
\hline Synechococcus elongatus PCC 7942 & - & - \\
\hline Synechococcus elongatus PCC 6301 & - & - \\
\hline Prochlorothrix hollandica PCC 9006 & + & - \\
\hline Leptolyngbya sp. PCC 6406 & + & - \\
\hline Nodosilinea nodulosa PCC 7104 & + & - \\
\hline Synechococcus sp. PCC 7335 & + & - \\
\hline Leptolyngbya sp. PCC 7375 & + & 4 \\
\hline Geitlerinema sp. PCC 7407 & + & 1 \\
\hline Leptolyngbya boryana PCC 6306 & + & 1 \\
\hline Thermosynechococcus elongatus BP-1 & - & - \\
\hline Synechococcus sp. PCC 6312 & - & - \\
\hline Cyanothece sp. PCC 7425 & - & - \\
\hline Acaryochloris marina MBIC11017 & - & - \\
\hline Acaryochloris sp. CCMEE 5410 & - & - \\
\hline Synechococcus sp. PCC 7502 & - & - \\
\hline Pseudanabaena sp. PCC 6802 & - & - \\
\hline Pseudanabaena biceps PCC 7429 & - & - \\
\hline Pseudanabaena sp. PCC 7367 & - & - \\
\hline Synechococcus sp. JA-3-3Ab & - & - \\
\hline Synechococcus sp. PCC 7336 & - & - \\
\hline Gloeobacter violaceus PCC 7421 & - & - \\
\hline
\end{tabular}

Figure S2. Co-occurrence of nsiR1 and hetF. Genomes are arranged and color-coded as in (Shih et al., 2013) as follows: filamentous non-heterocystous strains in green, heterocystous ramified strains in blue, heterocystous nonramified strains in pink, baeocystous strains in orange and unicellular strains in black. Nostoc sp. PCC 7120 is indicated in red. Presence or absence of a hetF homolog is indicated. Presence or absence of homologs of nsiR1, number of putative $n s i R 1$ copies and genomic location of the $n s i R 1$ arrays upstream to hetF (light green) or in a different genomic region (yellow) are also indicated. 


\section{A}

NSIR1.1 GGUAGAUGCACCUUGAUCUUUAACUCCCCUAGUUGGUUGAUAGCCACUGGGGGUUUUUU-NsiR1.4 GGUAGAUGCACCCUGAUAACUAACUCCCCUAGCUGGCUAACACCGACUGGGGGCUUUUUUU

B

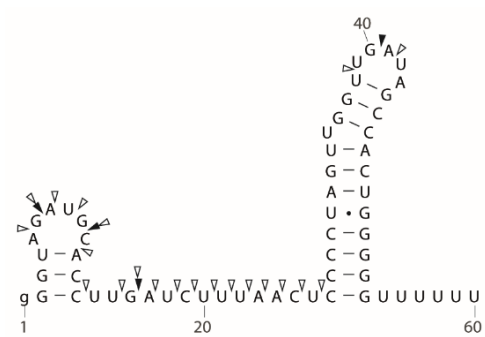

NsiR1.1

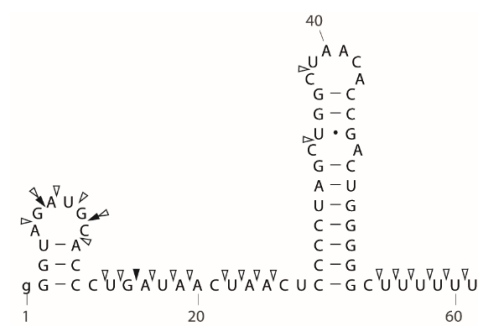

NsiR1.4

C

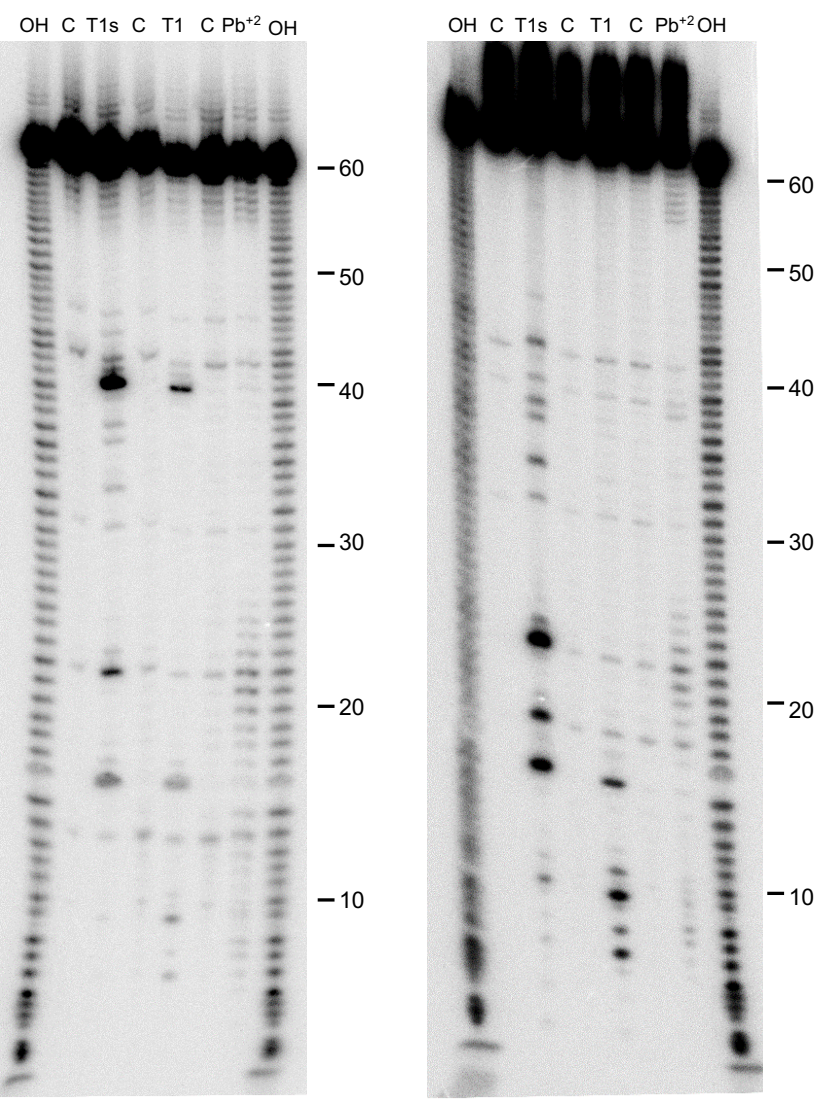

Figure S3. Sequence and structure probing of NsiR1.1 and NsiR1.4. A. Comparison of the sequences of NsiR1.1 and NsiR1.4. Nucleotide differences are highlighted in red. B. Secondary structure model of NsiR1.1 and NsiR1.4. Black and withe triangles indicate the positions sensitive to hydrolysis by RNase T1 or lead(II) acetate $\left(\mathrm{Pb}^{+2}\right)$, respectively. The guanosine added at the $5^{\prime}$ end for T7 transcription efficiency is in lower case. C. 5'-end labelled NsiR1.1 (left) or NsiR1.4 (right) were incubated with RNase T1 or lead(II) acetate and the resulting fragments analyzed on an $8 \%$ polyacrylamide sequencing gel. $\mathrm{C}$, untreated control; OH, alkaline ladder; T1s, RNase T1 ladder. Nucleotide positions of NsiR1.1 or NsiR1.4 are shown on the right. 


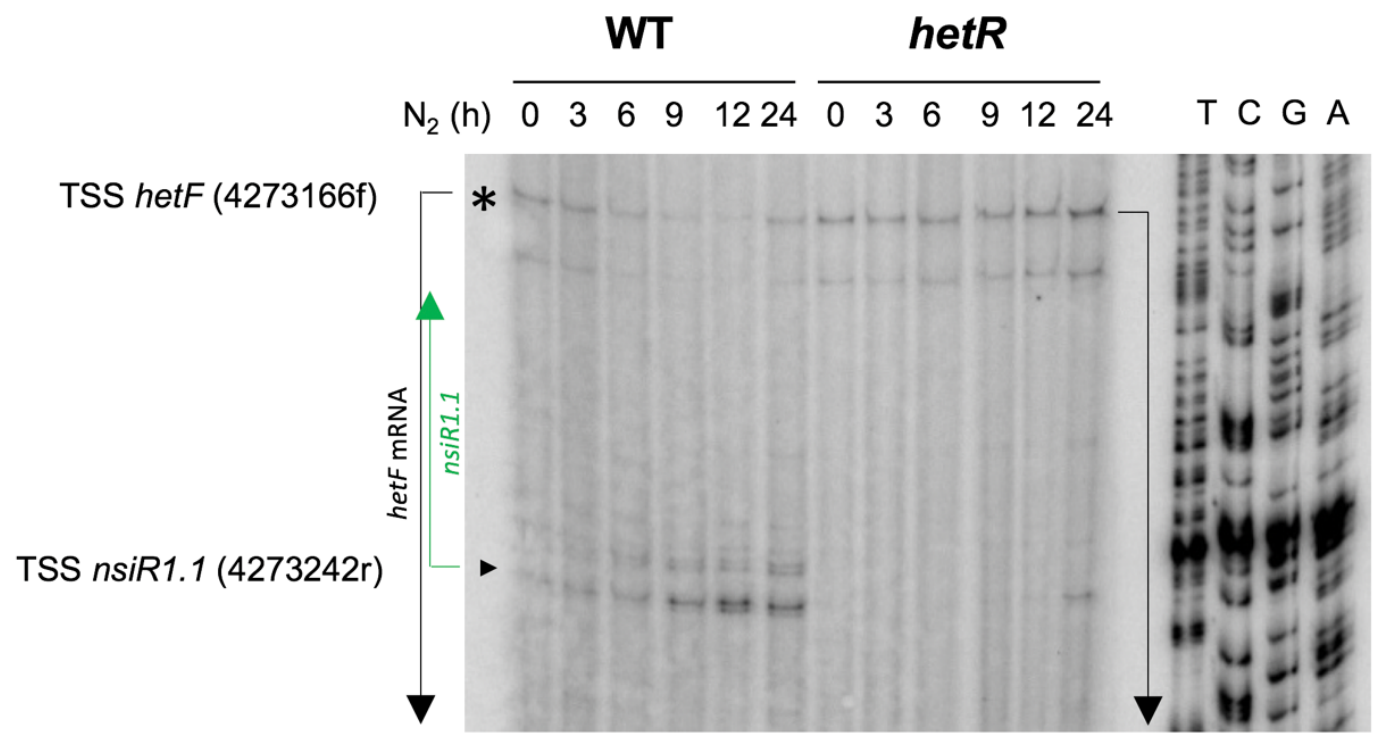

Figure S4. Primer extension analysis of the hetF transcript. Total RNA was isolated from Nostoc sp. PCC 7120 wild type strain (WT) or mutant strain 216 (hetR) at different times after removal of combined nitrogen. Primer extension assays were carried out using an oligonucleotide complementary to the 5 ' UTR of hetF. The position of the TSS of hetF (4273166f) is indicated with an asterisk. The position corresponding to the TSS of $n \operatorname{siR} 1.1$ (4273242r) is indicated with a black triangle. On the right, a sequencing reaction performed with the same oligonucleotide used for primer extension is included. The relative position of hetF (black arrow) and nsiR1.1 transcripts (green arrow) is indicated. 
A

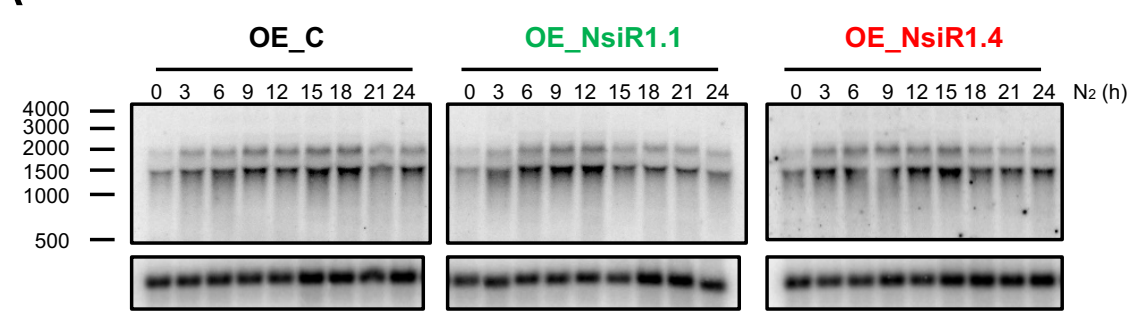

B

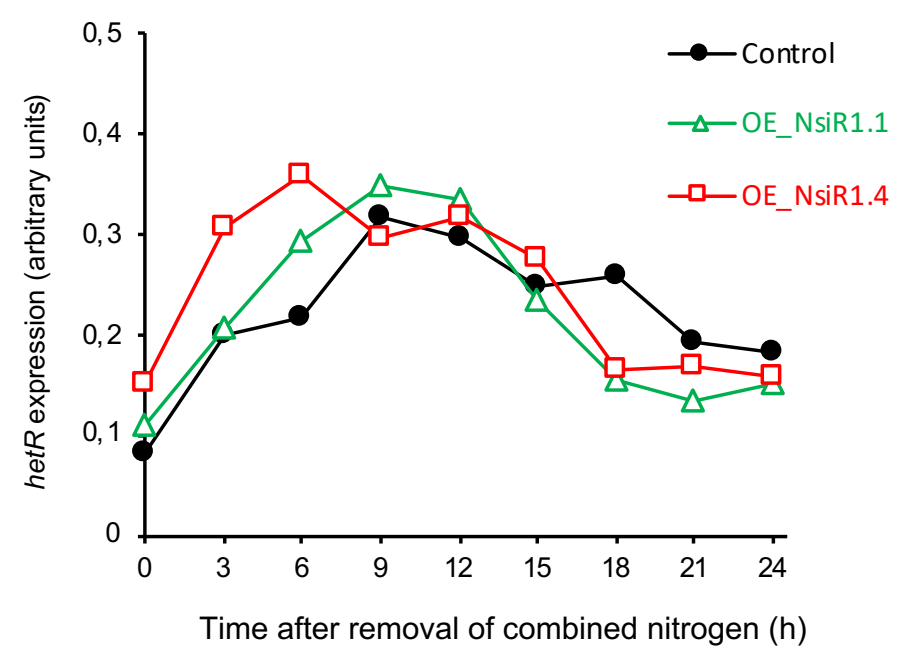

Figure S5. Accumulation of hetR transcripts in OE_C, OE_NsiR1.1, OE_NsiR1.4 and OE_as_NsiR1 strains. A. hetR expression. Northern blots with RNA from the indicated strains at different time points (indicated in hours) after removal of combined nitrogen were hybridized with probes for het $R$ (top panels) and $r n p B$ (bottom panels), used as a loading control. Membranes are the same used in Fig 4. B. Quantification of hetR expression. The data are presented as hetR/rnpB expression. 
Lyngbya majuscula 3L \#1

Crinalium epipsammum PCC 9333 \#1

Microcoleus sp. PCC 7113 \#1

Microcoleus chthonoplastes PCC 7420 \#1

Leptolyngbya sp. PCC 7375 \#1

Leptolyngbya sp. PCC 7375 \#2

Leptolyngbya sp. PCC 7375 \#

Leptolyngbya sp. PCC 7375 \#4

Leptolyngbya sp. PCC 6306 \#

Calothrix sp. PCC 7103 \#1

Nostoc sp. PCC 7120 \#12

Anabaena variabilis ATCC 29413 \#12

Chlorogloeopsis sp. PCC 7702 \#2

Fischerella sp. JSC-11 \#2

Fischerella sp. PCC $9431 \# 5$
Fischerella sp. PCC $9431 \# 2$

Fischerella sp. PCC 9339 identical \#4-5

Fischerella sp. PCC 9339 identical \#2-3

Fischerella sp. PCC 9431 identical \#3-4

Fischerella sp. JSC-11 \#3

Fischerella sp. PCC 9605 identical \#1 and \#3

Fischerella sp. PCC 9605 identical \#2 and \#4

Fischerella sp. PCC 9431 \#6

Calothrix sp. PCC 7103 \#2

Anabaena cylindrica PCC 7122 \#

Anabaena sp. PCC 7108 \#

Cylindrospermum stagnale PCC 7417 identical \#2-7

Anabaena cylindrica PCC 7122 \#9

Nostoc azollae 0708 \#7

Cylindrospermopsis raciborskii CS505 \#3

Anabaena sp. PCC 7108 identical \#4-5

Anabaena sp. PCC 7108 \#

Anabaena sp. PCC 7108 \#

Anabaena Cylindrica PCC 7122 identical \#4-5

Nostoc azollae 0708 \#2

Anabaena cylindrica PCC 7122 \#6

Anabaena cylindrica PCC 7122 \#3

Nostoc azollae 0708 identical \#3-5

Nostoc azollae 0708 \#6

Cylindrospermopsis raciborskii CS505 \#2

Nostoc sp. PCC 7120 \#11

Anabaena variabilis ATCC 29413 \#11

Nostoc sp. PCC 7524 \#3

Nodularia spumigena CCY9414 \#10

Nostoc sp. PCC 7524 \#11

Calothrix sp. PCC 6303 identical \#3-7

Calothrix sp. PCC 6303 \#8

Mastigocladopsis repens PCC 10914 identical \#2-4

Calothrix sp. PCC $6303 \# 2$

Scytonema hofmanni PCC 9009 \#2

Nostoc punctiforme PCC 73102 \#7

Nostoc punctiforme PCC 73102 \#8

Calothrix sp. PCC 7103 \#6

PCC 7103 \#8

sp. PCC $7116 \quad \# 1$

Nostoc punctiforme PCC 73102 \#3

Nostoc punctiforme PCC 73102 \#

Nodularia spumigena CCY9414 \#1

Nodularia spumigena CCY9414 identical \#2-8

Cylindrospermum stagnale PCC 7417 \#9

Cylindrospermum stagnale PCC 7417 \#10

Nostoc punctiforme PCC 7310236

Nostoc sp. PCC 7107 identical \#2-1

Nostoc sp. PCC 7120 identical \#3-9

Anabaena variabilis ATCC 29413 identical \#3-9

Nostoc sp. PCC 7524 identical \#4-9

Microchaete sp. PCC 7126 identical \#2-9

Calothrix sp. PCC 7507 \#3

Microchaete sp. PCC $7126 \# 10$

Calothrix sp. PCC 7507 \#2

Calothrix sp. PCC 7103 \#3

Calothrix sp. PCC $6303 \# 1$

Rivularia sp. PCC 7116 identical \#3-5 and \#7-12

Rivularia sp. PCC $7116 \# 6$
Rivularia sp. PCC 7116 \#2

Rivularia sp. PCC 7116 \#2
Cylindrospermum stagnale PCC 7417 \#1

Fischerella sp. JSC-11 \#1

Fischerella sp. PCC 9339 \#1

Mastigocladopsis repens PCC $10914 \# 1$

Mastigocladopsis repens
Anabaena sp. PCC 7108 \#1

Anabaena sp. PCC 7108 \#1 7122 \#1

Nostoc azollae 0708 \#1

Microchaete sp. PCC 7126 \#1

Calothrix sp. PCC 7507 \#

Nostoc sp. PCC 7524 \#1
Nostoc punctiforme PCC 73102 \#1

Nostoc punctiforme PCC
Nostoc sp. PCC 7107 \#1

Nostoc sp. PCC 7107 \#1

Cylindrospermopsis raciborskii CS505 \#

Nostoc sp. PCC $7120 \# 1$

Anabaena variabilis ATCC 29413 \#1

Scytonema hofmanni PCC 9009 \#1

Calothrix sp. PCC 7103 \#4

Nostoc sp. PCC 7524 \#2

Calothrix sp. PCC 7103 \#7
GGUAGAUGCACCCU-GAUUGACA-C---CCCCG-GUUCACUUUG-GUGAGC-UGGGG-AUUU

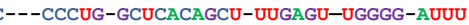
GGUAGAUGCACCCU-GAUUGACGUU---CCCUG-GCUCACCGGU-UUGAGU-CGGGG-AUUU GGUAGAUGCACCCU-GAUUGACGUU---CCCUG-GCUCACUGAU-UUGGGC-UAGGG-AUUU GGUAGAUGCACCCU-GAUGUCUAAC---UCCUG-AGCCCUU-CU-GGGACU-UGGGA-GCUU GGUAGAUGCACCCU-GAUGUCUGAC---UCCUA-AGCCCUU-UU-UGGGCC-UGGGA-GUUU GGUAGAUGCACCCU-GAUGUCUGAC---UCCUA-AGCCCUU-CU-GGGGUU-UGGGA-GUUU GGUAGAUGCACCCU-GAUGUCUGAC---UCCUG-AGCCCUU-UU-GGGGCU-UGGGA-GUUU GGUAGAUGCACCCU-GAUUUAU-U---GCCCUCGUCCAUGCCUAGUGGAC-AGGGC-UUUU GGUAGAUGCACCUU-AAUCUUCAGU---CCCCC-AAUUGUUUAAAAUCAAAU-GGGG-UUUU GGUAGAUGCACCUC-AAUAACUAAC-UCCCUAGUUUGGUUAAGA-CUAA-CUGGUG-AUUG GGUAGAUGCACCUC-AAUAACUAAC-UCCCUAGUUUGGUUAAUA-CUAA-CUGGUG-AUUG AGUAGAUACACCCU-GAUAACUUACAUUCCCCUAGUUAACUGACU-GUUAC-UAGGGG-AUUC GGUAGAUGCACCCU-GAUAACUUGC-UCCCCUAGUUAACUGUCG-GUUGC-UAGGGG-AUUU GGUAGAUGCACCCU-GAUAACUCGC-UCCCCUAGUUAACUGUCG-GUUGC-UAGGGG-AUUU GGUAGAUGCACCCU-GAUAACUCGC-UCCCCUUGAUAGCUGUCG-GCUAC-UUGGGG-AUUU GGUAGAUGCACCCU-GAUAACUCGC-UCCCCUUGAUAGCUGCUG-GCUAC-UAGGGG-AUUU GGUAGAUGCACCCU-GAUAACAAAC-UCCCCUUGAUAGCUGUCG-GCUAC-UAGGGG-AUUU GGUAGAUGCACCCU-GAUAACAAAC-UCCCCUUGAUAGCUGUCG-GCUAC-UUGGGG-AUUU GGUAGAUGCACCCU-GAUAACAAAC-UCCCCUUGCUAGCUGUCG-GCUAU-CGGGGG-AUUU GGUAGAUGCACCCU-GAUAACAGAC-UCCCCUUAAUAGCUGCCG-GCUAC-GAGGGG-DUUU GGUAGAUGCACCCU-GAUAACAGAC-UCCCCUUACUAGCUGUCG-GCUAC-GAGGGG-UUUU GGUAGAUCCACCCU-GAUAACAMACGGUAGAUGCACCCU-GAUAACAAAC-ACCCCUAGUUGACUGUCG-GUUGC-UAGGGG-AUUU GGUAGAUGCACCCU-GAUAAUAACC-UCCCCUAGUUGGAAACUU-UCCAC-UAGGGG-UUCU GGUAGAUGCACCCU-GAUAACUAAU-UUCCCCUGGUAGGCUAACA-CCCAC-UGGGGG-UUUU GGUAGAUGCACCCU-GAUUACUAAA-CUCCCCUGGCUGGCUAACA-CUCAC-UAGGGG-UUUU GGUAGAGCACCU-GAUAACAAA-CUCCCCCGUUGGCUAACA-CCCAU-UGGGGG-GUUU GGUAGAUGCACCU-GAUAACUAA-UUCCCCGACUGCOAAAA-CCUAC-UGGGGG-UUUU GGUAGAUGCACCCU-GAUAACUAAU-CUCCCCUGGAUGCCAACA-CCUAC-UAGGGG-UUUU GGUAGAUGCACCCU-GAUACUAAG-CUCCCCUAGAUGGCUAAUR-CCUAC-UGGGGG-UOUU AGUAGAUGCACCCU-GAUAACUAAC-CUCCCCUAGCUGGCUAACA-C-CUC-UGGGGG-UUUU GGUAGAUGCACCCU-GAUAACUAAU-CUCCCCUAGCUGGCUAACA-C-CUC-UGGGGG-GUUU GGUAGAUGCACCCU-GAUAACUAAU-CUCCCCUAGCUGGCUAACA-C-CUC-UGGGGG-UUUU AGUAGAUGCACCCU-GAUAACUAAC-UUCCCCUAGCUGGCUAACA-C-CAC-UGGGGG-UUUU GGUAGAUGCACCCU-GAUAACUAAU-CUCCCCUAGCUGGCUAACA-C-CAC-UGGGGG-UUUU AGUAGAUGCACCCU-GAUAACUAAC-UUCCCCUAGCUGGCUAACA-C-CAC-UUGGGG-UUUU GGUAGAUGCACCCU-GAUAAUUAAU-UUCCCCUAGUUGGCUAACA-C-CAC-UGGGGG-UUUU AGUAGAUGCACCCU-GAUAACUAAU-UUCCCCUAGUUGGCUAACA-C-CGC-UGGGGG-UUUU AGUAGAUGCACCCU-GAUAACUAAU-CUCCCCUAGUGGGCUAACA-C-CAC-UAGGGC-UUUU GGUAGAUGCACCCU-GAUAAUUAAU-CUCCUCCGG-AGGCCUUAA-GCCUC-UGGGGG-UUUU GGUAGAUGCACCCU-GAUAACUAA-CUCCCCUAGUUGGCUGCCA-C-CAC-UAGGGG-UUUU GGUAGAUGCACCCU-GAUAACUAA-CUCCCCUAGCUGGCUGCCA-C-CAC-UAGGGG-UUUU GGUAGAUGCACCCU-GAUAACUAA-CUCCCCUGGCUGGCUGCCA-C-CAC-CGGGGG-UUUU GGUAGAUACACACU-GAUAACUAA-CUCCCCUGGCUGGCUGCCA-C-CAC-UAGGGG-UUUU GGUAGAUGCACCCUAAAUAAUUGC-CUCCCCUGGUUGGCUGCCA-C-CAC-UAGGGG-CUGU GGUAGAUGCACCCU-GAUAACU-U-CUUCCCUAGCU-GGCUAACACUGGC-UGGGGG-AUUU GGUAGAUGCACCCU-GAUAACUCAU-UUCCCUUAGCU-AAUUGAUUUUGGC-UGGGGG-AUUU GGUAGAUGCACCCU-GAUAACUCAU-UUCCCUUAGCU-GAUUAAUUUCGGC-UGGGG-ACUU GGUAGAUGCACCCU-GAUAACUCAC-UCCCCUGGUCGGCUUACCAGCUGC-UUGGGG-AUUU GGUAGAUGCACCCU-GAUAACCCAC-UCCCUUAGCC-GAUUGCCAUCAGC-UGAGGG-AUUU GGUAGAUGCACCCU-GAUAACUCAC-UUUCCCCUGGUUGGUUGCCACCCCC-UGGGGGGAGAOCACCUGGUAGAGCACCUU-GAUAACUAAC-UCCCCUAUAGACAACA-UCAUC-UGGGGG-UUUU GGUAAUCACCU-GAUAAUAC-UCCCUAGURGA GGUAGUGCACCU-GAOACUCAC-UCCCOAGUAGAOCCA-UCCGC-UAGGGG-UUUU GGUAGAUGCACCCU GGUAGAUGCACCCU-GAUAACUAAC-UCCCCUGGUUGACUGCCU-UCCAC-UAGGGG-UUUU GGUAGAUGCACCCU-GAUAACUAGC-UCCCCUGGUUGGCUAACA-CCCAC-UGGGGG-UUUU AGUAGAUGCACCCU-GAUAACUAAC-UCCCCUGACUGGCUAACA-CCCGU-UGGGGG-AUUU AGUAGAUGCACCCU-GAUAACUAAC-UCCCCUGACUGGCUAACA-CCCGU-UGGGGG-AUUU GGUAGAUGCACCCU-GAUAACUAA-UCCCCUGACUGGCUAACA-CCCGU-UGGGGG-AUUU GGUAGAUGCACCCU-GAUAACUAAC-UCCCCCCGUUGGCUAACA-CCCGC-UGGGGG-UUUU AAUAGAUGCACCCU-GAUAACUUAC-UCCCCUGGUUGGCUAAAA-CCCAC-UGGGGG-UUUU GGUAGAUGCACCCU-GAUAACUAAU-UCCCCUAGCUGGUUUACA-CCGAC-UGGGGG-AUUU GGUAGAUGCACCCU-GAUAACUAAC-UCCCCUAGCUGGCUAACA-CCGAC-UGGGGG-CUUU GGUAGAUGCACCCU-GAUAACUAAC-UCCCCUAGCUGGCUAACA-CCGAC-UGGGGG-CUUU GGUAGAUGCACCCU-GAUAACUAAC-UCCCCUGGUUGGCUAACACCUGAC-UAGGGG-CUUU GGUAGAUGCACCCU-GAUAACUAAC-UCCCCUGGUAGGACGAUU-CCUGC-UGGGGG-AUUU GGUAGAUGCACCCU-GAUAACUAAC-UUCCCUGGUAGGACAACU-CUUAC-UAGGGA-AUUU GGUAGAUGCACCCU-GAUAACUAAC-UCCCCUAGUAGGACGACU-UCUAC-UGGGGG-GGCU GGUAGAUGCACCCU-GAUAACUAAC-UCCCCUGGUAGGACAACU-CCUAC-UAGGGU-UUUU GGUAGAUGCACCCU-GAUAACUCAC-UCCCUUAGCUGUUUAAUU-ACGGC-UAAGGG-UUUU GGUAGAUGCACCCU-GAUAACUCAU-UUCCCUAGUUGGCUUAAG-CUAAC-UAGGGU-UUUU GGUAGAUGCACCCU-GAUAACUAAC-UCCCCCGGUAAGCCU-ACAAGCACG-CUGGGG-GAUU GGUAGAUGCACCCU-GAUAACUGUC-UCCCCCGGUAAGCCU-ACAAGCACG-CUGGGG-GAUU GGUAGAUGCACCCU-GAUAAAUAAC-UCCCCCGGUAAGCCU-ACGAGCACG-CUGGGG-GAUU GGUAGAUGCACCUU-GAUCUUCAAC---UCCCCUGCUUGGCUAACAGCCAC-AGGGGU-UUOU GGUAGAUGCACCUU-GAUCUUUAAC---UCCCCUGCUUGGCUAAUACACGC-UGGGGG-GUUU GGUAGAUGCACCUU-GAUCUUUAAC----UCCCCUGUUUGGCUAAUACACAC-UGGGGA-GUUU GGUAGAUGCACCUU-GAUCUUUAAC---UCCCCUGUUUGGCUAAUACACUC-UGGGGA-GUUU GGUAGAUGCACCUU-GAUCUUC-AA---CUCCCAUGUAGGCUAACACCUUC-UGGGAG-UUUU GGUAGAUGCACCUU-GAUCUUCAAA-U-CCCCUGGUAGG-UUAACACCCGCCCAGGG-UUU GGUAGAUGCACCUU-GAUCUUCAAA-U-CCCCUGGUAGG-UUAACACCCGCCCAGGGG-UUU GGUAGAUGCACCUU-GAUCUUCAAC-U-CCCCUGGUAGG-CUAACACCCGC-CGGGGG-UUU GGUAGAUGCACCUU-GAUCUUCAAC-U-CCUCUGGUAGG-UUAACACCCAC-CGGAGG-UUU GGUAGAUGCACCUU-GAUCUUCGAC-U-CCUCUGGUUGG-UGAAAGCCCGC-CAGAGG-AUUU GGUAGAUGCACCUU-GAUCUUCGAC-U-CCUCUGGUUGG-UGAGAGCCUGC-CAGAGG-AUUU GGURA GGUAGAUGCACCUU-GAUCUUCAAC-U-CCCCUAGUUGG-CUAACACCUAC-UAGGGG-UUUU GGUAGAUGCACCUU-GAUCUOCAAC-UCCCCOAGCUGG-UUAACACCGC-UGGGG-AUUU GURG UGCACCUU-G UCUUCAAC-U-CCCCUARCUGG-UURUGCGCCUU-URGGGG-GUUU GGUAGAUGCACCUU-GAUCUUCAAC-U-CCCCUAGCUGG-CUGCAGGCCUC-UAGGG-UUUU GGUAGAUGCACCUU-GAUCUUUAAC-U-CCCCUAGUUGG-UUGAUAGCCAC-UGGGGG-UUUU GGUAGAUGCACCUU-GAUCUUCAAU-UCCCCCGGUUGGC-UUUACACCCAAGUGGGG-GUUU

GGUAGAUGCACCCU-GAUAACCCAC-UCCCCUAAUGGACUAAAU-GUCUAAUUAGGG-GUUU GGUGAAUACACCCU-GAUAAAUAAC-UUCCCUAGUUGGCUAAAA-CCUG-ACUAGGG-GCUU GGUAGAUGCACCCU-GAUAACUCA-----CUCCCUAGUAGAUCUCCAUCCGCUAGG-GUUUUU 
Spirulina subsalsa PCC 9445 \#1

Raphidiopsis brookil D9 2

Nostoc punctiforme PCC 73102 \#4

Cylindrospermum stagnale PCC 7417 \#8

Nodularia spumigena CCY9414 \#1

Nostoc sp. PCC 7524 \#12

Mastigocladopsis repens PCC 10914 \#5

Anabaena cylindrica PCC 7122 \#8

Nodularia spumigena CCY9414 \#9

Nostoc sp. PCC 7524 \#10

Microchaete sp. PCC 7126 \#11

Oscillatoria acuminata PCC 6304 \#1

Oscillatoria acuminata PCC 6304 \#2

Geitlerinema sp. PCC 7407 \#1

Oscillatoria sp. PCC 10802 \#1

Oscillatoria sp. PCC 10802 \#2

Arthrospira sp. PCC 8005 \#1

Trichodesmium erythraeum IMS101 \#1

Geitlerinema sp. PCC 7105 \#1

Oscillatoria sp. PCC 6506 \#1

OsCillatoria sp. PCC 6506 \#2

Microcoleus vaginatus FGP-2 \#4

Sccillatoria nigroviridis PCC 7112 \#2

Microcoleus vaginatus FGP-2 \#2

Microcoleus vaginatus FGP-2 \#2

Oscillatoria nigroviridis PCC 7112 \#1

Microcoleus vaginatus $\mathrm{FCP}-2$ \#1

Nostoc punctiforme PCC 73102 \#5

Chlorogloeopsis sp. PCC 7702 \#1

Nostoc punctiforme PCC 73102 \#2

Anabaena sp. PCC 7108 \#2
GGUAGAUGCACCCU-GAUAGCUCU-----CCCAUGUCAUUUUCCCUGAAGUGGCAUGGGAUUUU GGUAGAUGCACCCU-GAUAACUAAAUCUCCCCCUAGUCGGACAAAACCGCU-GGGGG-UUUU GGUAGAUCCACCCU-GAUACUUAAC-UCCCUGAAUUAGCUAACA-CUUUC-UUGGAG-AUUU GAUA GAUGCACCCU-GAUAA GGUAGAUGCACCUU-GAUAACUAAC-UCUCUUAGCUGUCUAACACCCAAU---GGGGCUUUU AGUAGAUCCACCCU-GAUACUAC-UCCCUUUU GGUAAAUGCACCGU-GAUAACUAAC-UCCCACUUUUAGUUCAUACUCAUU---GGAGUAUUU GGUAGAUCACCU-GAUACOAC-UCCACUUAGUCAUACUCAUU---GGAGUAUUU GCUAUAUUUCACCCU-GAUAACUAAC-UUCCCCUAGCUGACCAACACUCUC-UGAGAG-UUUU GAUAAAUGCGUU-GAUAACUAAC-UUCCCCUAGCUGACCAACACUCUC-UGAGA-UUUU GAUGAAUGAACCCU-AAUCAAUAAC-UCCCCUAAUUGGCUGCCA---CGGAUAGGAG-CUUU UGUAGAUGCACCU-AAUCAAUAAC-UCCCCUAAUUGGCUGCCA---CGGAUAGGAG-CUUU GGUAGAUGCACCCU-GAUAACUGAC---UCCUCGGUUAGACCAA-ACGCC-GGGA-A-UUU GGUAGAUGCACCCU-GAUUGAC-G----UUUCCCGGCUCUUUU----GAGCC-GGUU-U-UUU GGUAGAUGCACCCU-GAUUAAU-U----UCCCCAGC-CCAC-C---CGGAC-UGGGGA-UUU GGUAGAUGCACCCU-GAUUGAU-G-----GUCUCGGCUUUAU-C---CUGGC-CGGGAU-UUU GGUAGAUGCACCCU-GAUGAAC-------UUCCCCGCCCGAC-C---CGGGC-GGGGUU-UUU AGUAGAUGCACCCU-GAUGGUU------UUCCCCUGGCUUC------GGCUG-GGGGAU-UUU AGUAGAUGCACCCU-GAUA-UA-C----GACCUCCACCGUCAU---AGGUG-GGGGUU-UUU GGUAGAUGCACCCU-GAUAGUU-A-----ACCCCGGCACGACAC---CCAGC-CGGGGU-UUU AGUAGAUGCACCCU-GAUGGAUAA-----CC-CCCAACCAAUUC---UGG-C-UGGGGU-UUU AGUAGAUGCACCCU-GAUAGAUGA----AC-CCUAACCAAUUA---CGG-C-UAGGGU-UUU AGUAGAUGCACCCU-GAUUAAUAA----AC-CCCAGCCAAUUU---UGG-C-CGGGCU-UUU AGUAGAUGCACCCU-GAUUAAAUA-----ACUCCCGACCAAUUU---UGG---UGGGGU-UUU GGUAGAUGCACCCU-GAUUAAAUA-----ACUCCCGACCAAUUU---UGG---CCGGGU-UUU AGUAGAUGCACCCU-GAUUAAUUA--AGUAGAGCACCO-GAUAAOA-GGURUGCACCCU-GAUUAACA----ACUCCCGACCAAUU-OGUU--OGGU-UUU GUAAUCACOU GAOU GAACCA CO UCU-GAUACUAAC-UCCUCUGGUUGUUAACAACU---DUGAGGUUUU GAUROU GAUAGAUGUCCCUU-GAUUUUUAAC-UCCCCUAAUUCAGUUAAUAUCUAC-UAGAG-UUUC

Figure S6. Alignment of 132 repeats of nsiR1 generated with mLocARNA (Will et al., 2012). Each repeat is represented by the name of the organism and the number of the repeat. Identical copies of $n$ siR 1 for each genome are included only once and marked "identical". nsiR1.1 homologs that might be transcribed antisense to hetF 5'UTR in heterocystous cyanobacteria are framed in purple. Nucleotides are shown in different colors in order to facilitate visualization of the alignment. 


\section{Supplementary tables}

\section{Table S1. Strains}

\begin{tabular}{|c|c|c|}
\hline Strain & Description & Reference \\
\hline \multicolumn{3}{|l|}{ Escherichia coli } \\
\hline $\mathrm{DH} 5 a$ & Used for routine transformation & (Hanahan, 1983) \\
\hline \multicolumn{3}{|l|}{ Nostoc sp. } \\
\hline PCC 7120 & Wild type & $\begin{array}{l}\text { Pasteur Culture } \\
\text { Collection }\end{array}$ \\
\hline 216 & Non-functional HetR. S179N mutation. & $\begin{array}{l}\text { (Buikema and } \\
\text { Haselkorn, 1991) }\end{array}$ \\
\hline OE_C & $\begin{array}{l}\mathrm{Sm}^{\mathrm{R} S p^{\mathrm{R}}} \text {, pMBA51 inserted in plasmid alpha. T1 } \\
\text { terminator of } E \text {. coli rrnB gene expressed } \\
\text { constitutively from the trc promoter. }\end{array}$ & $\begin{array}{l}\text { (Olmedo-Verd et } \\
\text { al., 2019) }\end{array}$ \\
\hline OE_NsiR1.1 & $\begin{array}{l}\mathrm{Sm}^{\mathrm{R} S p} \mathrm{R}^{\mathrm{R}} \text {, pMBA77 inserted in plasmid alpha. } \\
\text { NsiR1.1 transcribed constitutively from the trc } \\
\text { promoter. }\end{array}$ & This work \\
\hline OE_NsiR1.4 & $\begin{array}{l}\mathrm{Sm}^{\mathrm{R} S p^{\mathrm{R}}} \text {, pMBA78 inserted in plasmid alpha. } \\
\text { NsiR1.4 expressed constitutively from trc promoter. }\end{array}$ & This work \\
\hline OE_as_NsiR1 & $\begin{array}{l}\mathrm{Sm}^{\mathrm{R} S p^{\mathrm{R}}}, \mathrm{pMBA} 42 \text { inserted in plasmid alpha. } \\
\text { Antisense to NsiR1.1 transcribed constitutively from } \\
\text { the trc promoter. }\end{array}$ & This work \\
\hline
\end{tabular}


Table S2. Plasmids

\begin{tabular}{|c|c|c|}
\hline Name & Description & Reference \\
\hline pJV300 & $\begin{array}{l}\text { Control plasmid expresses a } \sim 50 \text { nt nonsense RNA derived } \\
\text { from the } T 1 \text { terminator of } E \text {. coli } r r n B \text { gene. }\end{array}$ & $\begin{array}{l}\text { (Sittka et al., } \\
\text { 2007) }\end{array}$ \\
\hline pXG0 & $\mathrm{Cm}^{\mathrm{R}}$, control plasmid without GFP & $\begin{array}{l}\text { (Urban and } \\
\text { Vogel, 2007) }\end{array}$ \\
\hline pXG10-SF & $\begin{array}{l}\mathrm{Cm}^{\mathrm{R}} \text {, plasmid for construction of translational sfGFP fusions, } \\
\text { under control of the PLteto-1 promoter. }\end{array}$ & $\begin{array}{l}\text { (Corcoran et } \\
\text { al., 2012) }\end{array}$ \\
\hline pZE12-luc & $\begin{array}{l}\mathrm{Ap}^{R} \text {, plasmid used for the expression of NsiR1 under control of } \\
\text { the PLlac-o promoter. }\end{array}$ & $\begin{array}{c}\text { (Lutz and } \\
\text { Bujard, 1997) }\end{array}$ \\
\hline pRL278 & $\begin{array}{l}\mathrm{Nm}^{\mathrm{R}} \text {, shuttle vector for conjugation in Nostoc bearing a } \\
\text { Neomycin resistance gene. }\end{array}$ & $\begin{array}{l}\text { (Black et al., } \\
\text { 1993) }\end{array}$ \\
\hline pSAM270 & $\begin{array}{l}\text { Plasmid bearing a transcriptional fusion of the hetR promoter } \\
\text { to gfpmut2 }\end{array}$ & $\begin{array}{l}\text { (Brenes- } \\
\text { Álvarez et al., } \\
\text { 2019) }\end{array}$ \\
\hline pMBA20 & $\begin{array}{l}A^{R} \mathrm{Sm}^{\mathrm{R} S p^{R}} \text {, plasmid for the overexpression of transcripts from } \\
\text { the } r n p B \text { promoter and followed by the } T 1 \text { transcriptional } \\
\text { terminator of } E \text {. coli rrnB gene. }\end{array}$ & $\begin{array}{l}\text { (Olmedo-Verd } \\
\text { et al., 2019) }\end{array}$ \\
\hline pMBA37 & 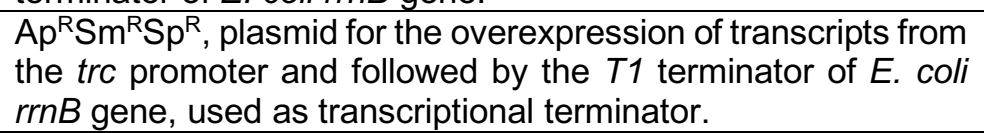 & $\begin{array}{l}\text { (Olmedo-Verd } \\
\text { et al., 2019) }\end{array}$ \\
\hline pMBA51 & 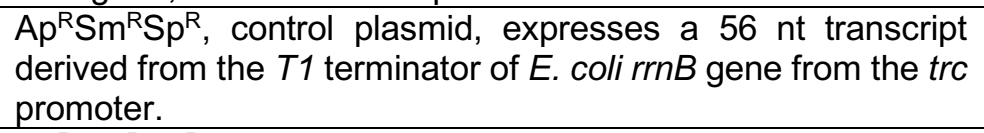 & $\begin{array}{l}\text { (Olmedo-Verd } \\
\text { et al., 2019) }\end{array}$ \\
\hline pMBA42 & $\begin{array}{l}\mathrm{Ap}^{R} \mathrm{Sm}^{\mathrm{R} S p^{\mathrm{R}}} \text {, plasmid based on pMBA37 for } \\
\text { overexpression of an antisense to NsiR1.1. }\end{array}$ & This work \\
\hline pMBA77 & $\begin{array}{l}\mathrm{Ap}^{R} \mathrm{Sm}^{\mathrm{R}} \mathrm{Sp}^{\mathrm{R}} \text {, plasmid based on pMBA37 for the } \\
\text { overexpression of NsiR1.1. }\end{array}$ & This work \\
\hline pMBA78 & $\begin{array}{l}\mathrm{Ap}^{\mathrm{R}} \mathrm{Sm}^{\mathrm{R} S p^{\mathrm{R}}} \\
\text { overexpression of NsiR 1.4. }\end{array}$ & This work \\
\hline pMBA91 & $\begin{array}{l}\mathrm{Ap}^{\mathrm{R}} \mathrm{Nm}^{\mathrm{R}} \text {, Kanamycin/ Neomycin resistance version of } \\
\text { pMBA20. }\end{array}$ & This work \\
\hline pMBA92 & $\begin{array}{l}\mathrm{Ap}^{\mathrm{R}} \mathrm{Nm}^{\mathrm{R}} \text {, plasmid based on pMBA91 expressing from the } r n p B \\
\text { promoter the gene gfpmut2 plus its 5'UTR. }\end{array}$ & This work \\
\hline pMBA93 & $\begin{array}{l}\mathrm{Ap}^{\mathrm{R}} \mathrm{Nm}^{\mathrm{R}} \text {, plasmid based on pMBA91 expressing from the } r n p B \\
\text { promoter a translational fusion of hetF } 5 \text { 'UTR plus sequences } \\
\text { encoding the first } 20 \text { amino acids of HetF fused to GFP. }\end{array}$ & This work \\
\hline pMML2 & $\begin{array}{l}\mathrm{Cm}^{\mathrm{R}} \text {, sfGFP reporter plasmid based on pXG10-SF containing } \\
\text { the hetF 5'UTR plus sequences encoding the first } 20 \text { amino } \\
\text { acids of HetF (with mutation in start codon GTG -> ATG). }\end{array}$ & This work \\
\hline $\mathrm{pMML3}$ & $A p^{R}$, plasmid based on pZE12-luc expressing NsiR1.1. & This ! \\
\hline pMML4 & $A p^{R}$, plasmid based on pZE12-luc expressing NsiR1.4. & This work \\
\hline
\end{tabular}


Table S3. Oligonucleotides

\begin{tabular}{|c|c|c|}
\hline Name & Sequence (5'-3') & Used for \\
\hline 105 & CGCCAAAGTTTCAATTCGATTTCC & Primer extension of hetF \\
\hline $\begin{array}{l}189 \\
\text { (PLlacOB) }\end{array}$ & CGCACTGACCGAATTCATTAA & \multirow{2}{*}{$\begin{array}{l}\text { Plasmid backbone } \\
\text { amplification from pZE12- } \\
\text { luc }\end{array}$} \\
\hline $\begin{array}{l}190 \\
\text { (PLlacOD) }\end{array}$ & GTGCTCAGTATCTTGTTATCCG & \\
\hline 506 & GTTTTGAGCTCAGGTCTAGGGCGGCGGATTT & $\begin{array}{l}\text { Probes for } n \operatorname{siR} 1.1 \text { and } \\
n s i R 1.4\end{array}$ \\
\hline 512 & $\begin{array}{l}\text { TTTTATCTCGAGGTAGATGCACCCTGATAACTAACTC } \\
\text { CCCTAGCTGGCTAACACCGACTGGGGGCTTTTTATGC } \\
\text { ATGCTCCAGTGTAATC }\end{array}$ & $\begin{array}{l}\text { Cloning of as_nsiR1 } \\
\text { fused to the trc promoter } \\
\text { in pMBA37 }\end{array}$ \\
\hline 555 & $\begin{array}{l}\text { GTTTTATGCATAAGTATATGCTAGTTATAGAAAAAAC } \\
\text { CCCC }\end{array}$ & \multirow{4}{*}{$\begin{array}{l}\text { Cloning of hetF 5'UTR } \\
\text { with mutation in start } \\
\text { codon (GTG->ATG) in } \\
\text { pXG10-SF }\end{array}$} \\
\hline 556 & GAAATTCCTGGGACATAACTTCTCCTATC & \\
\hline 557 & GTTTTGCTAGCCCGCACCAAGTAGTCATTCTGCC & \\
\hline 558 & GATAGGAGAAGTTATGTCCCAGGAATTTC & \\
\hline 559 & 5'P-GGTAGATGCACCTTGATCTTTAA & \multirow{2}{*}{$\begin{array}{l}\text { Cloning of } n \operatorname{siR} 1.1 \text { in } \\
\text { pZE12-luc } \\
\end{array}$} \\
\hline 561 & GTTTTTTCTAGACCCAAGACAGTAGAATAATCTAAG & \\
\hline 560 & 5'P-GGTAGATGCACCCTGATAACTA & \multirow{2}{*}{$\begin{array}{l}\text { Cloning of } n s i R 1.4 \text { in } \\
\text { pZE12-luc }\end{array}$} \\
\hline 562 & GTTTTTTCTAGACGGATGAATCTGCACAATGATG & \\
\hline 573 & GTTTTATCGATGCGCCGACATCATAACGGTT & \multirow{2}{*}{$\begin{array}{l}\text { Cloning of the trc } \\
\text { promoter }\end{array}$} \\
\hline 574 & GTTTTATGCATTCCACACATTATACGAGCCG & \\
\hline 757 & $\begin{array}{l}\text { GTTTTATCTCGAGAAAAAACCCCCAGTGGCTATCAAC } \\
\text { CAACTAGGGGAGTTAAAGATCAAGGTGCATCTACCAT } \\
\text { GCATTCCACACATTATACG }\end{array}$ & $\begin{array}{l}\text { Cloning of } n s i R 1.1 \text { fused } \\
\text { to the trc promoter in } \\
\text { pMBA37 }\end{array}$ \\
\hline 758 & $\begin{array}{l}\text { GTTTTATCTCGAGAAAAAGCCCCCAGTCGGTGTTAGC } \\
\text { CAGCTAGGGGAGTTAGTTATCAGGGTGCATCTACCAT } \\
\text { GCATTCCACACATTATACG }\end{array}$ & $\begin{array}{l}\text { Cloning of } n s i R 1.4 \text { fused } \\
\text { to the trc promoter in } \\
\text { pMBA37 }\end{array}$ \\
\hline 787 & GTACTGCAAGGGGCGTGGCT & \multirow[t]{2}{*}{ Probe for $n i f H$} \\
\hline 788 & ССTATTGGTAGCTTCTGCGGG & \\
\hline 831 & $\begin{array}{l}\text { TAATACGACTCACTATAGGGAAGTATATGCTAGTTAT } \\
\text { AGAAAAAACCCCC }\end{array}$ & \multirow{2}{*}{$\begin{array}{l}\text { PCR of template for in } \\
\text { vitro transcription of hetF } \\
\text { 5'UTR }\end{array}$} \\
\hline 873 & CCGCACCAAGTAGTCATTCTG & \\
\hline 833 & $\begin{array}{l}\text { TAATACGACTCACTATAGGGTAGATGCACCTTGATCT } \\
\text { TTAACTCCCCTAGTTGGTTG }\end{array}$ & \multirow{2}{*}{$\begin{array}{l}\text { PCR of template for in } \\
\text { vitro transcription of } \\
\text { NsiR1.1 }\end{array}$} \\
\hline 834 & $\begin{array}{l}\text { AAAAAACCCCCAGTGGCTATCAACCAACTAGGGGAGT } \\
\text { TAAAGATC }\end{array}$ & \\
\hline 835 & $\begin{array}{l}\text { TAATACGACTCACTATAGGGTAGATGCACCCTGATAA } \\
\text { CTAACTCCCCTAGCTGGCTAAC }\end{array}$ & \multirow{2}{*}{$\begin{array}{l}\text { PCR of template for in } \\
\text { vitro transcription of } \\
\text { NsiR1.4 }\end{array}$} \\
\hline 836 & $\begin{array}{l}\text { AAAAAAGCCCCCAGTCGGTGTTAGCCAGCTAGGGGAG } \\
\text { TTAG }\end{array}$ & \\
\hline hetR8 & GCGCTCTGGTGGCACATCG & \multirow[t]{2}{*}{ Probe for hetR } \\
\hline hetR9 & CATGAGGACGAGCGGGCATA & \\
\hline 892 & GTTTTGGATCCCTGCATCCCTTAACTTACTTAT & \multirow{2}{*}{$\begin{array}{l}\text { Cloning of Nm resistance } \\
\text { cassette in pMBA20 }\end{array}$} \\
\hline 893 & GTCTAGAGGATCCCCGGTGGG & \\
\hline 894 & GTTTTCTCGAGGCATGCCTGCAGGTCTGGACAT & $\begin{array}{l}\text { Cloning of translational } \\
\text { fusions to GFP in } \\
\text { pMBA91 }\end{array}$ \\
\hline 895 & СТССTTTACTCCGCACCAAGTAGTCATTCTGC & \multirow{2}{*}{$\begin{array}{l}\text { Cloning of hetF 5'UTR } \\
\text { fused to GFP }\end{array}$} \\
\hline 896 & TACTTGGTGCGGAGTAAAGGAGAAGAACTTTTC & \\
\hline 899 & GTTTTATGCATGCGAAAGCTTGCATGCCTGCAGG & Cloning of gfpmut2 5'UTR \\
\hline
\end{tabular}

Restriction sites used for cloning (underlined), point mutations made in hetF start codon (red) and 5' monophosphate (5'P) are indicated. The T7 promoter sequence in oligonucleotides 831,833 and 835 is in bold. 
Table S4. Sequences of inserts in plasmids expressing GFP translational fusions in Nostoc.

\begin{tabular}{|c|c|c|}
\hline Plasmid & Sequence & Description \\
\hline pMBA92 & 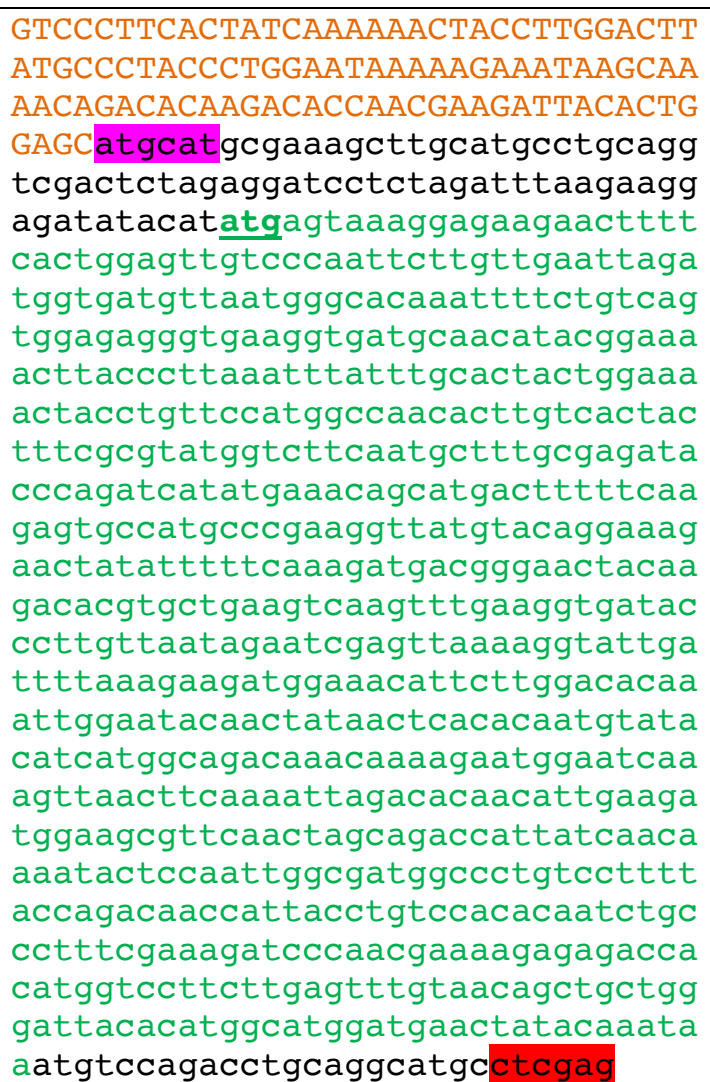 & $\begin{array}{l}\text { gfpmut2 plus its original 5'UTR } \\
\text { downstream the } r n p B \text { promoter }\end{array}$ \\
\hline pMBA93 & 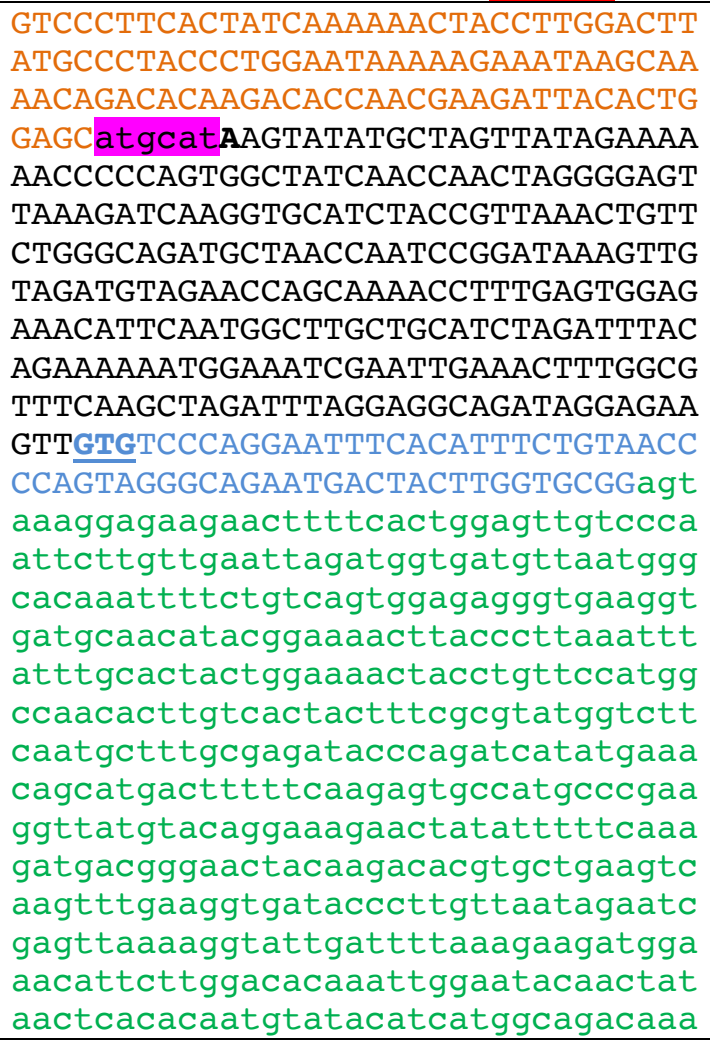 & $\begin{array}{l}\text { Translational fusion of hetF } \\
5 \text { 'UTR plus sequences encoding } \\
\text { the first } 20 \text { amino acids of HetF } \\
\text { fused to GFP, downstream the } \\
\text { rnpB promoter }\end{array}$ \\
\hline
\end{tabular}




\begin{tabular}{|l|l|l|}
\hline caaagaatggaatcaaagttaacttcaaatt \\
agacacaacattgaagatggaagcgttcaacta \\
gcagaccattatcaacaaatactccattggc \\
gatggcctgtcctttaccagacaaccattac \\
ctgtccacacatctgcctttcgaagatccc \\
aacgaaagagagaccacatggtccttcttgag & \\
tttgtaacagctgctgggattacacatggcatg & \\
gatgaactatacaaataatgtccagacctgca & \\
ggcatgctcgag & \\
\hline
\end{tabular}

Nostoc sequences are capitalized, with black letters corresponding to 5'UTR, blue letters corresponding to hetF coding sequences and orange letters corresponding to the $r n p B$ promoter, respectively. Nsil and Xhol sites that were used for cloning are highlighted in magenta and red, respectively. Start codons are shown in bold and underlined. The coding sequence of gfpmut2 is in green. 
Table S5. RNAs used for in vitro footprinting assays.

\begin{tabular}{|c|c|}
\hline Name & Sequence (5'-3') \\
\hline NsiR1.1 & $\begin{array}{l}\text { gGGUAGAUGCACCUUGAUCUUUAACUCCCCUAGUUGGUUGAUAGCCACUGGGGG } \\
\text { UUUUUU }\end{array}$ \\
\hline NsiR1.4 & $\begin{array}{l}\text { gGGUAGAUGCACCCUGAUAACUAACUCCCCUAGCUGGCUAACACCGACUGGGGG } \\
\text { CUUUUUU }\end{array}$ \\
\hline hetF 5'UTR & $\begin{array}{l}\text { gggAAGUAUAUGCUAGUUAUAGAAAAAACCCCCAGUGGCUAUCAACCAACUAGG } \\
\text { GGAGUUAAAGAUCAAGGUGCAUCUACCGUUAAACUGUUCUGGGCAGAUGCUAAC } \\
\text { CAAUCCGGAUAAAGUUGUAGAUGUAGAACCAGCAAAACCUUUGAGUGGAGAAAC } \\
\text { AUUCAAUGGCUUGCUGCAUCUAGAUUUACAGAAAAAAUGGAAAUCGAAUUGAAA } \\
\text { CUUUGGCGUUUCAAGCUAGAUUUAGGAGGCAGAUAGGAGAAGUUGUGUCCCAGG } \\
\text { AAUUUCACAUUUCUGUAACCCCAGUAGGGCAGAAUGACUACUUGGUGCGGUUCU } \\
\text { GUAACCCCAGUAGGGCAGAAUGACUACUUGGUGCGG }\end{array}$ \\
\hline
\end{tabular}

Nostoc sequences are capitalized, guanosines added for T7 transcription efficiency are in lower case. Predicted interaction site of hetF 5'UTR with different versions of NsiR1 is highlighted in yellow. Start codon is shown in bold and underlined. 
Table S6. Sequences of inserts in plasmids used for verification of sRNAmRNA interactions in E. coli.

\begin{tabular}{|c|c|c|}
\hline Plasmid & Sequence & Description \\
\hline pMML2 & $\begin{array}{l}\text { atgcatAAGTATATGCTAGTTATAGAAAAAACCCCCAGTGGCTATC } \\
\text { AACCAACTAGGGGAGTAAAGATCAAGGTGCATCTACCGTTAAACT } \\
\text { GTTCTGGGCAGATGCTAACCAATCCGGATAAAGTTGTAGATGTAGA } \\
\text { ACCAGCAAAACCTTGAGTGGAGAAACATTCAATGGCTTGCTGCAT } \\
\text { CTAGATTTACAGAAAAAATGGAAATCGAATTGAAACTTTGGCGTTT } \\
\text { CAAGCTAGATTTAGGAGGCAGATAGGAGAAGTTATGTCCCAGGAAT } \\
\text { TTCACATTCTGTAACCCCAGTAGGGCAGAATGACACTTGGTGCG } \\
\text { ggctagc }\end{array}$ & hetF 5'UTR \\
\hline pMML3 & $\begin{array}{l}\text { GGTAGATGCACCTTGATCTTTAACTCCCCTAGTTGGTTGATAGCCA } \\
\text { CTGGGGGTTTTTTCTATAACTAGCATATACTTAGATTATTCTACTG } \\
\text { TCTTGGGtctaga }\end{array}$ & NsiR1.1 \\
\hline pMML4 & $\begin{array}{l}\text { GGTAGATGCACCCTGATAACTAACTCCCCTAGCTGGCTAACACCGA } \\
\text { CTGGGGGCTTTTTTATGTTCATAAATAACCAGCATCATTGTGCAGA } \\
\text { TTCATCCGTCAGATCTAGAtctaga }\end{array}$ & NsiR1.4 \\
\hline
\end{tabular}

Nostoc sequences are capitalized, with black letters corresponding to 5'UTR and blue letters corresponding to coding sequences, respectively. Nsil and Nhel sites that were used for cloning are highlighted in magenta and yellow, respectively. Nucleotide changed with respect to wild type sequence is indicated in red. Grey shadowed letters indicate the NsiR1.1 and NsiR1.4 sequences. Xbal restriction sites used for cloning are highlighted in blue. The start codon of hetF is shown in bold and underlined. 


\section{Supplementary references}

Black, T.A., Cai, Y., and Wolk, C.P. (1993) Spatial expression and autoregulation of hetR, a gene involved in the control of heterocyst development in Anabaena. Mol Microbiol 9: 77-84.

Brenes-Álvarez, M., Mitschke, J., Olmedo-Verd, E., Georg, J., Hess, W.R., Vioque, A., and Muro-Pastor, A.M. (2019) Elements of the heterocyst-specific transcriptome unravelled by co-expression analysis in Nostoc sp. PCC 7120. Environ Microbiol 21: 2544-2558.

Buikema, W.J., and Haselkorn, R. (1991) Characterization of a gene controlling heterocyst differentiation in the cyanobacterium Anabaena 7120. Genes Dev 5: 321-330.

Corcoran, C.P., Podkaminski, D., Papenfort, K., Urban, J.H., Hinton, J.C., and Vogel, J. (2012) Superfolder GFP reporters validate diverse new mRNA targets of the classic porin regulator, MicF RNA. Mol Microbio/ 84: 428-445.

Hanahan, D. (1983) Studies on transformation of Escherichia coli with plasmids. J Mol Biol 166: 557-580.

Lutz, R., and Bujard, H. (1997) Independent and tight regulation of transcriptional units in Escherichia coli via the LacR/O, the TetR/O and AraC//1-I2 regulatory elements. Nucleic Acids Res 25: 1203-1210.

Olmedo-Verd, E., Brenes-Álvarez, M., Vioque, A., and Muro-Pastor, A.M. (2019) A heterocyst-specific antisense RNA contributes to metabolic reprogramming in Nostoc sp. PCC 7120. Plant Cell Physiol 60: 1646-1655.

Shih, P.M., Wu, D., Latifi, A., Axen, S.D., Fewer, D.P., Talla, E. et al. (2013) Improving the coverage of the cyanobacterial phylum using diversity-driven genome sequencing. Proc Natl Acad Sci USA 110: 1053-1058.

Sittka, A., Pfeiffer, V., Tedin, K., and Vogel, J. (2007) The RNA chaperone Hfq is essential for the virulence of Salmonella typhimurium. Mol Microbio/ 63: 193-217.

Urban, J.H., and Vogel, J. (2007) Translational control and target recognition by Escherichia coli small RNAs in vivo. Nucleic Acids Res 35: 1018-1037.

Will, S., Joshi, T., Hofacker, I.L., Stadler, P.F., and Backofen, R. (2012) LocARNA-P: accurate boundary prediction and improved detection of structural RNAs. RNA 18: 900-914. 
Figure S1

Trichodesmium erythraeum IMS101

Arthrospira sp. PCC 8005

Oscillatoria nigroviridis PCC 7112

Microcoleus vaginatus FGP-2

Oscillatoria sp. PCC 6506

Oscillatoria sp. PCC 10802

Oscillatoria acuminata PCC 6304

Fischerella sp. PCC 9605

Fischerella sp. PCC 9431

Fischerella sp. PCC 9339

Fischerella sp. JSC-11

Chlorogloeopsis sp. PCC 7702

Mastigocladopsis repens PCC 10914

Calothrix sp. PCC 7103

Calothrix sp. PCC 6303

Nostoc sp. PCC 7524

Nostoc sp. PCC 7120

Anabaena variabilis ATCC 29413

Nostoc sp. PCC 7107 region1

Nostoc sp. PCC 7107 region2

Cylindrospermum stagnale PCC 7417

Anabaena cylindrica PCC 7122

Anabaena sp. PCC 7108

Raphidiopsis brookii D9

Cylindrospermopsis raciborskii CS505

Nostoc azollae 0708

Nodularia spumigena CCY9414

Calothrix sp. PCC 7507

Microchaete sp. PCC 7126

Nostoc punctiforme PCC 73102

Scytonema hofmanni PCC 9009

Rivularia sp. PCC 7116

Spirulina subsalsa PCC 9445

Lyngbya majuscula 3L

Microcoleus chthonoplastes PCC 7420

Microcoleus sp. PCC 7113

Crinalium epipsammum PCC 9333

Geitlerinema sp. PCC 7105

Leptolyngbya sp. PCC 7375

Geitlerinema sp. PCC 7407

Leptolyngbya sp. PCC 6306

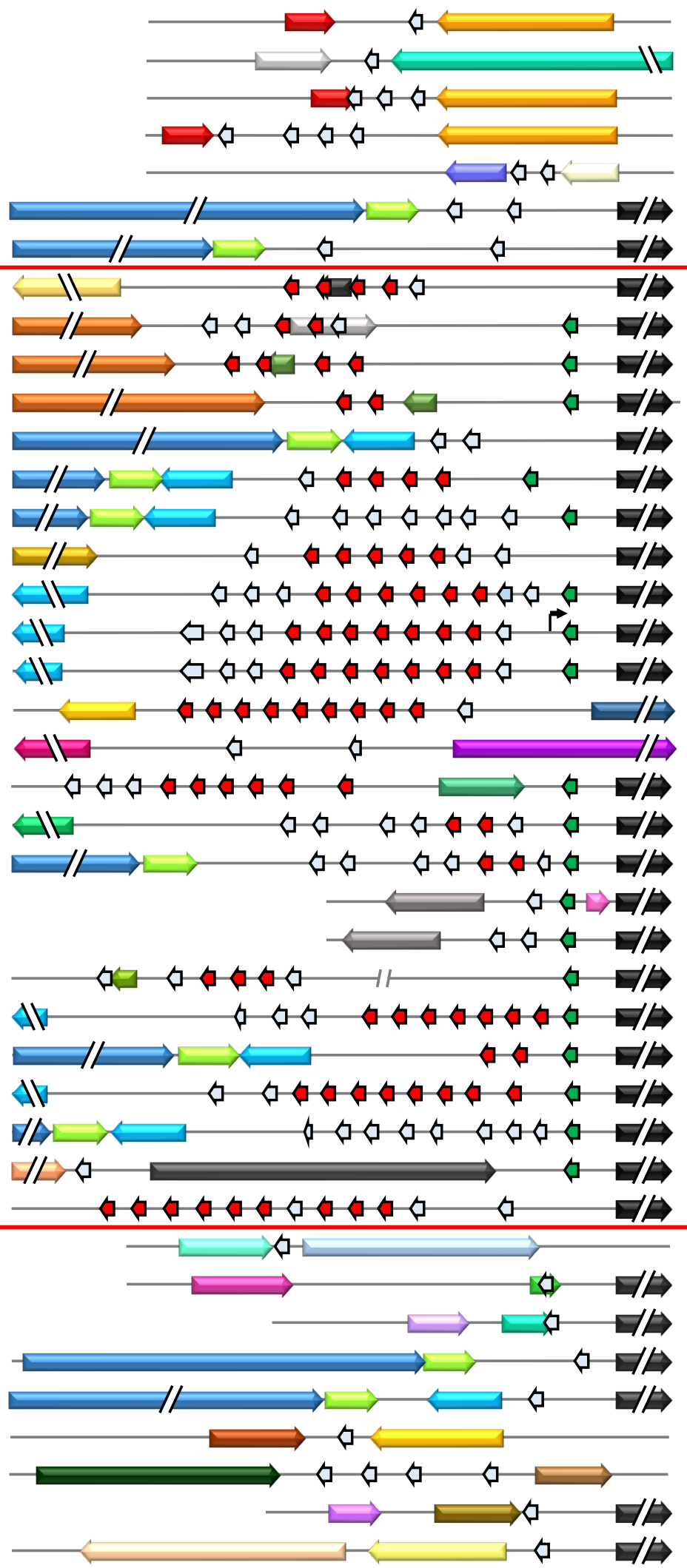




\section{Figure S2}

\begin{tabular}{|c|c|c|}
\hline Organism & hetF & nsiR1 \\
\hline Trichodesmium erythraeum IMS101 & + & 1 \\
\hline Arthrospira platensis str. Paraca & + & - \\
\hline Arthrospira platensis NIES-39 & + & - \\
\hline Arthrospira sp. PCC 8005 & + & 1 \\
\hline Arthrospira maxima CS-328 & + & - \\
\hline Oscillatoria nigroviridis PCC 7112 & + & 3 \\
\hline Microcoleus vaginatus FGP-2 & + & 4 \\
\hline Oscillatoria sp. PCC 6506 & + & 2 \\
\hline Oscillatoria formosa PCC 6407 & + & - \\
\hline Oscillatoria sp. PCC 10802 & + & 2 \\
\hline Oscillatoria acuminata PCC 6304 & + & 2 \\
\hline Fischerella sp. 9605 & + & 5 \\
\hline Fischerella sp. PCC 9431 & + & 6 \\
\hline Fischerella sp. PCC 9339 & + & 5 \\
\hline Fischerella sp. PCC JSC-11 & + & 3 \\
\hline Cyanobacterium PCC 7702 & + & 2 \\
\hline Mastigocladopsis repens PCC 10914 & + & 6 \\
\hline Calothrix sp. PCC 7103 & + & 8 \\
\hline Calothrix sp. PCC 6303 & + & 8 \\
\hline Nostoc sp. PCC 7524 & + & 12 \\
\hline Nostoc sp. PCC 7120 & + & 12 \\
\hline Anabaena variabilis ATCC 29413 & + & 12 \\
\hline Nostoc sp. PCC 7107 & + & 13 \\
\hline Cylindrospermum stagnale PCC 7417 & + & 10 \\
\hline Anabaena cylindrica PCC 7122 & + & 8 \\
\hline Anabaena sp. PCC 7108 & + & 8 \\
\hline Raphidiopsis brookii D9 & + & 2 \\
\hline Cylindrospermopsis raciborskii CS-505 & + & 3 \\
\hline Nostoc azollae 0708 & + & 7 \\
\hline Nodularia spumigena CCY 9414 & + & 11 \\
\hline Calothrix sp. PCC 7507 & + & 3 \\
\hline Microchaete sp. PCC 7126 & + & 11 \\
\hline Nostoc punctiforme PCC 73102 & + & 9 \\
\hline Scytonema hofmanni UTEX 2349 & + & 2 \\
\hline Rivularia sp. PCC 7116 & + & 12 \\
\hline Synechocystis sp. PCC 7509 & - & - \\
\hline Gloeocapsa sp. PCC 7428 & - & - \\
\hline Chroococcidiopsis thermalis PCC 7203 & - & - \\
\hline Dactylococcopsis salina PCC 8305 & - & - \\
\hline Halothece sp. PCC 7418 & - & - \\
\hline Spirulina subsalsa PCC 9445 & + & 1 \\
\hline Spirulina major PCC 6313 & + & - \\
\hline Cyanothece sp. CCY0110 & - & - \\
\hline Cyanothece sp. ATCC 51472 & - & - \\
\hline Cyanothece sp. ATCC 51142 & - & - \\
\hline
\end{tabular}

\begin{tabular}{|c|c|c|}
\hline Organism & hetF & $n s i R 1$ \\
\hline Crocosphaera watsonii WH0003 & - & - \\
\hline Crocosphaera watsonii WH8501 & - & - \\
\hline Cyanothece sp. PCC 8802 & - & - \\
\hline Cyanothece sp. PCC 8801 & - & - \\
\hline Synechocystis sp. PCC 6803 & - & - \\
\hline Pleurocapsa sp. PCC 7327 & - & - \\
\hline Microcystis aeruginosa NIES-843 & - & - \\
\hline Cyanothece sp. PCC 7822 & - & - \\
\hline Cyanothece sp. PCC 7424 & - & - \\
\hline Gloeocapsa sp. PCC 73106 & - & - \\
\hline Synechococcus sp. PCC 7002 & - & - \\
\hline Leptolyngbya sp. PCC 7376 & + & - \\
\hline Cyanobacterium stanieri PCC 7202 & - & - \\
\hline Geminocystis herdmanii PCC 6308 & - & - \\
\hline Cyanobacterium aponinum PCC 10605 & - & - \\
\hline Stanieria cyanosphaera PCC 7437 & - & - \\
\hline Pleurocapsa sp. PCC 7319 & - & - \\
\hline Xenococcus sp. PCC 7305 & - & - \\
\hline Lyngbya majuscula $3 \mathrm{~L}$ & + & 1 \\
\hline Microcoleus chthonoplastes PCC 7420 & + & 1 \\
\hline Microcoleus sp. PCC 7113 & + & 1 \\
\hline Crinalium epipsammum PCC 9333 & + & 1 \\
\hline Chamaesiphon minutus PCC 6605 & - & - \\
\hline Geitlerinema sp. PCC 7105 & + & 1 \\
\hline Synechococcus elongatus PCC 7942 & - & - \\
\hline Synechococcus elongatus PCC 6301 & - & - \\
\hline Prochlorothrix hollandica PCC 9006 & + & - \\
\hline Leptolyngbya sp. PCC 6406 & + & - \\
\hline Nodosilinea nodulosa PCC 7104 & + & - \\
\hline Synechococcus sp. PCC 7335 & + & - \\
\hline Leptolyngbya sp. PCC 7375 & + & 4 \\
\hline Geitlerinema sp. PCC 7407 & + & 1 \\
\hline Leptolyngbya boryana PCC 6306 & + & 1 \\
\hline Thermosynechococcus elongatus BP-1 & - & - \\
\hline Synechococcus sp. PCC 6312 & - & - \\
\hline Cyanothece sp. PCC 7425 & - & - \\
\hline Acaryochloris marina MBIC11017 & - & - \\
\hline Acaryochloris sp. CCMEE 5410 & - & - \\
\hline Synechococcus sp. PCC 7502 & - & - \\
\hline Pseudanabaena sp. PCC 6802 & - & - \\
\hline Pseudanabaena biceps PCC 7429 & - & - \\
\hline Pseudanabaena sp. PCC 7367 & - & - \\
\hline Synechococcus sp. JA-3-3Ab & - & - \\
\hline Synechococcus sp. PCC 7336 & - & - \\
\hline Gloeobacter violaceus PCC 7421 & - & - \\
\hline
\end{tabular}

Figure S2. Co-occurrence of $n s i R 1$ and hetF. Genomes are arranged and color-coded as in (26) as follows: filamentous non-heterocystous in green, heterocystous ramified in blue, heterocystous non-ramified in pink, baeocystous in orange and unicellular in black. Nostoc sp. PCC 7120 is indicated in red. Presence or absence of a hetF homolog is indicated. Presence or absence of homologs of $n s i R 1$, number of putative $n s i R 1$ copies and genomic location of the $n s i R 1$ arrays upstream to hetF (light green) or in a different genomic region (yellow) are also indicated. 


\section{Figure S3}

\section{A}

NSIR1.1 GGUAGAUGCACCUUGAUCUUUAACUCCCCUAGUUGGUUGAUAGCCACUGGGGGUUUUUU-NsiR1.4 GGUAGAUGCACCCUGAUAACUAACUCCCCUAGCUGGCUAACACCGACUGGGGGCUUUUUUU

B

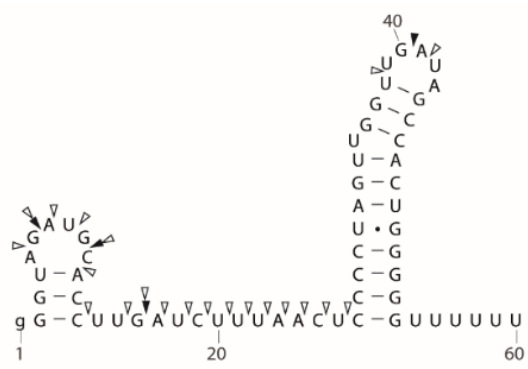

NsiR1.1

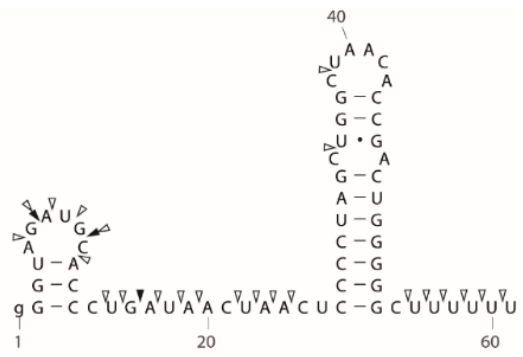

NsiR1.4

C

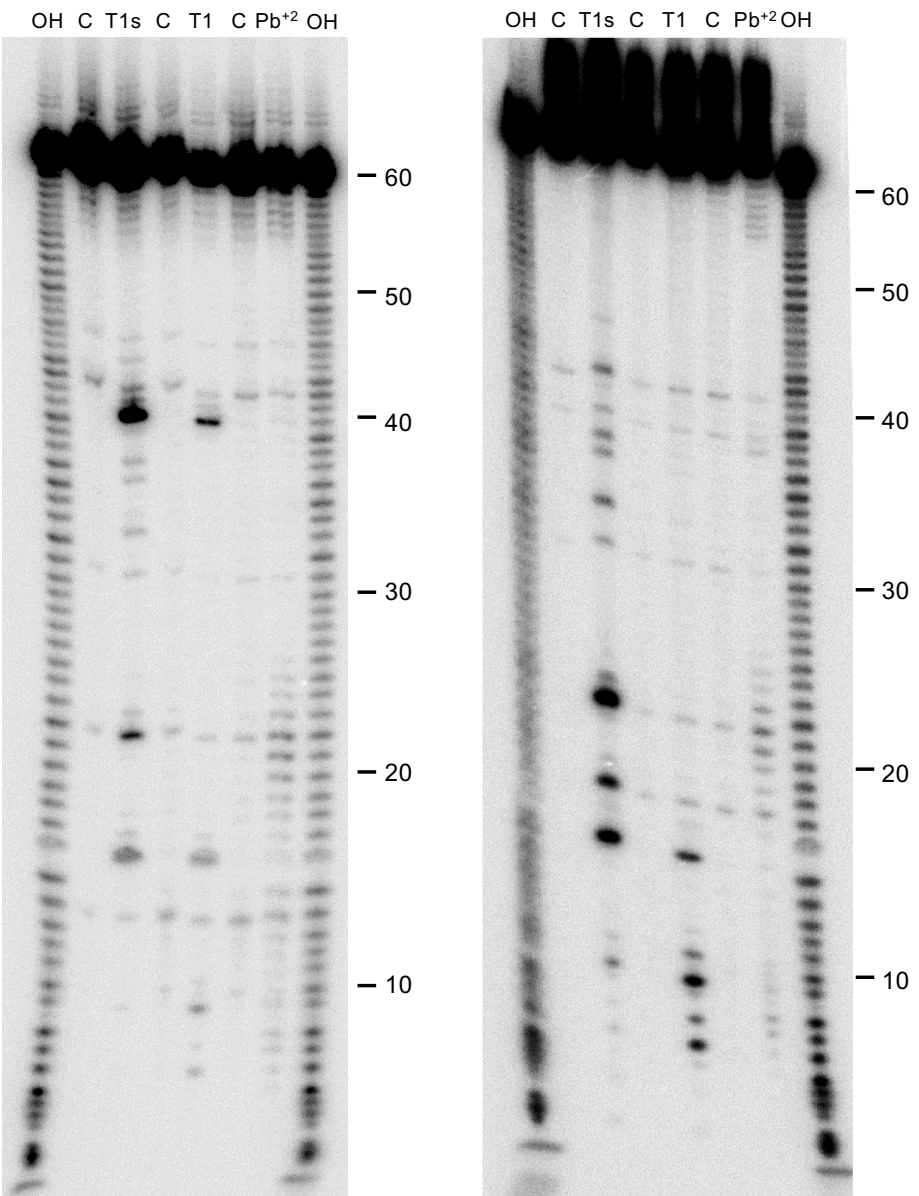

Figure S3. Sequence and structure probing of NsiR1.1 and NsiR1.4. (a) Comparison of the sequences of NsiR1.1 and NsiR1.4. Nucleotide differences are highlighted in red. The region involved in the interaction with alr3234 mRNA is underlined. (b) Secondary structure model of NsiR1.1 and NsiR1.4. Black and withe triangles indicate the positions sensitive to hydrolysis by RNase T1 or lead (II) acetate $(\mathrm{Pb})$, respectively. (c) 5'-end labelled NsiR1.1 (left) or NsiR1.4 (right) were incubated with RNase T1 or lead (II) acetate and the resulting fragments analyzed on a $8 \%$ polyacrilamide sequencing gel. $\mathrm{C}$, untreated control; $\mathrm{OH}$, alkaline ladder; $\mathrm{T} 1 \mathrm{~s}$, RNase T1 ladder. Nucleotide positions of NsiR1.1 or NsiR1.4 are shown on the left. 


\section{Figure S4}

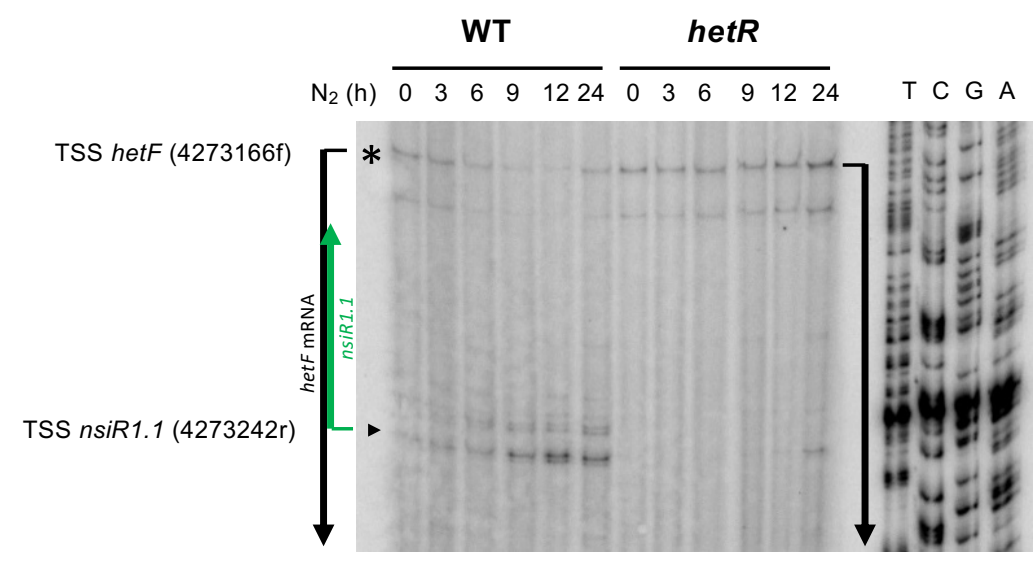

Figure S4. Primer extension analysis of the hetF transcript. Total RNA was isolated from Nostoc sp. PCC 7120 wild type strain (WT) or mutant strain 216 (hetR) at different times after removal of combined nitrogen. Primer extension assays were carried out using an oligonucleotide complementary to the 5' UTR of hetF. The position of the TSS of hetF (4273166f) is indicated with an asterisk. The position corresponding to the TSS of $n$ siR1.1 (4273242r) is indicated with a black triangle. On the right, a sequencing reaction performed with the same oligonucleotide used for primer extension is included. The relative position of hetF (black arrow) and nsiR1.1 RNAs (green arrow) is indicated. 


\section{Figure S5}

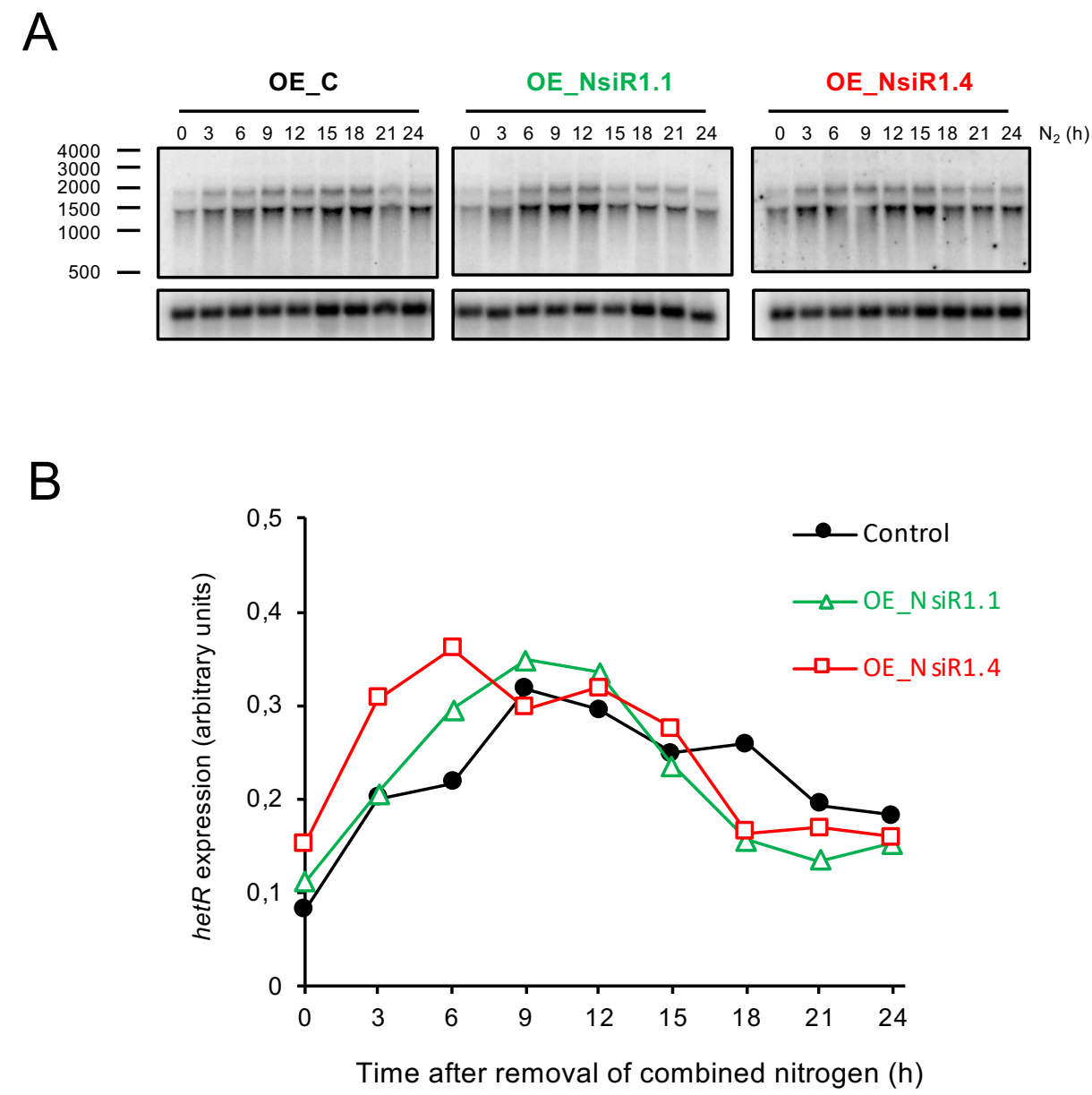

Figure S5. Accumulation of hetR transcripts in OE_C, OE_NsiR1.1, OE_NsiR1.4 and OE_as_NsiR1 strains. (a) hetR expression. Northern blots with RNA from the indicated strains at different time points (indicated in hours) after removal of combined nitrogen were hybridized with probes for hetR (top pannels) and $r n p B$ (bottom panels), used as a loading control. Membranes are the same used in Fig 7. (b) Quantification of hetR expression. The data is presented as hetR/rnpB expression. 